\title{
Sound sources in the interactions of two inviscid two-dimensional vortex pairs
}

\author{
By S. K. TA $\mathrm{NG}^{1}$ AND N. W. M. $\mathrm{KO}^{2}$ \\ ${ }^{1}$ Department of Building Services Engineering, The Hong Kong Polytechnic University, \\ Hong Kong, China \\ ${ }^{2}$ Department of Mechanical Engineering, The University of Hong Kong, Hong Kong, China
}

(Received 12 December 1998 and in revised form 12 April 2000)

The sources of sound during the interactions of two identical two-dimensional inviscid vortex pairs are investigated numerically by using the vortex sound theory and the method of contour dynamics. The sound sources are identified and then separated into two independent components, which represent the contributions from the vortex centroid dynamics and the microscopic vortex core dynamics. Results show that the sound generation mechanism of the latter is independent of the type of vortex pair interaction, while that of the former depends on the jerks, accelerations and vortex forces on the vortex pairs. The power developed by the vortex forces is found to be important in the generation of sound when the vortex cores are severely deformed and their centroids are close to each other. The isolated source terms also explain the appearance of wavy oscillations on the time variations of the sound source strengths in the vortex ring and the two-dimensional vortex interaction systems.

\section{Introduction}

It is well known that sound can be generated by the unsteady motion of vorticitybearing fluids, especially in low-Mach-number flows (Powell 1964; Howe 1975). Since coherent vortical structures are commonly found in turbulent shear layers (for instance, Davies 1973; Hussain 1986) and their interactions have been proved to be the major mechanism of shear layer growth (Winant \& Browand 1974), the study of sound generation by the motion of vorticity is important in understanding the noise produced.

There have been studies on the use of vortex models for investigating shear flows. Using the method of contour dynamics (see Pullin 1992), Shariff, Leonard \& Ferziger (1989) recovered the pairing of two inviscid vortex rings. In axisymmetric and plane shear layers, the coherent structures have been modelled as vortex rings and twodimensional vortex filaments respectively (Acton 1976). The use of vortex motion as a source of sound in the wave equation also gives rise to exact far-field solutions for a compact near field (Powell 1964; Möhring 1978). Leung et al. (1996) showed, using the theory of Möhring (1978), that the mutual threading of two identical inviscid vortex rings produces a sound field having features similar to those of the jet noise found experimentally (Lush 1971). When the source is not compact, exact solutions may still be found. Typical examples of sound generation by the unsteady motions of thin vortex filaments are those of Crighton (1972) and Tang \& Ffowcs Williams (1998).

The mechanism through which the vortices generate sound is of general interest in aeroacoustics. The vortex rings in axisymmetric jets, the vortex pairs in plane jets 
and the two-dimensional vortices in plane shear layers are expected to be the sources of aerodynamic noise (Crighton 1975). More recently, Powell (1995) proved that the inviscid vortex sound is produced through the second time derivatives of the vortex strength and vorticity moment. The results obtained by Tang \& Ko (1995), using the contour dynamics method and Möhring's theory, showed that the axial jerking and radial accelerating motion of the finite core vortex rings are important in the sound generation process. The deductions of Powell (1995) and Tang \& Ko (1995) are basically similar. The effect of the rate of change of the vortex strength, as described in Powell (1995), was implicitly included in the variations of the velocities, accelerations and jerks of the vortex ring discussed in Tang \& Ko (1995). The results of the interactions of two two-dimensional finite core vortices further confirm the importance of the accelerating motions of the vortices and the vortex-core deformation in the sound generation mechanism (Tang \& Ko 1997). The recent theory of Doak (1998) suggests that the fluctuating total enthalpy is the generalized acoustic field, and emphasizes the importance of the Coriolis acceleration of vorticity in affecting the propagation of this generalized pressure wave. However, the relationship between the Coriolis acceleration of vorticity and the dynamics of finite core vortices has not been addressed.

Extensive direct numerical simulations of the sound fields produced by shear flows have been done by Moin and his co-workers (for instance, Colonius, Lele \& Moin 1997; Mitchell, Lele \& Moin 1999). Though their results are in agreement with the theories, the source terms used are not related to the dynamics of the vortical structures in the shear flows.

Direct experimental investigation into the sound generation mechanism of coherent structures is, to the knowledge of the authors, rare. One remarkable comment from Laufer \& Yen (1983) is that the sound of a low-Mach-number circular jet is generated from a relatively small region, where high accelerations of the jet structures are found. Some direct experimental results relating the mean structure acceleration and subsonic jet sound generation can be found in Tang \& Ko (1993). However, the vortex motion that tends to generate substantial noise is still not clearly determined by experiments.

Vortex pairs are found in a two-dimensional jet of large aspect ratio (Beavers \& Wilson 1970). A vortex pair is two-dimensional and consists of two mirror-image-like vorticity patches with opposite-sign vorticity. Their interactions, such as leapfrogging motion and coalescence, resemble those of the vortex rings visualized by Yamada \& Matsui (1979). However, the vortex pairs are two-dimensional and thus the sound sources are non-compact. The aims of the present investigation are to study the sound fields created by the interactions of vortex pairs and to contribute to the understanding of the basic sound generation mechanism in finite-core vortex interactions. It is also hoped that the present results can provide a framework to examine more complex flows.

\section{Theories and computational considerations}

This section gives a brief discussion of the method of contour dynamics, the aeroacoustic theory used and the possible definitions of the centroid of a vortex pair. The sound field and the sound generation mechanism are also discussed.

\subsection{Contour dynamics method and vortex core centroids}

The method of contour dynamics is useful for the computation of the vortex core shapes during vortex pair interactions (Dritschel 1986). In this inviscid vortex model, vorticity is confined within the vortex cores. No vorticity will be found outside the 


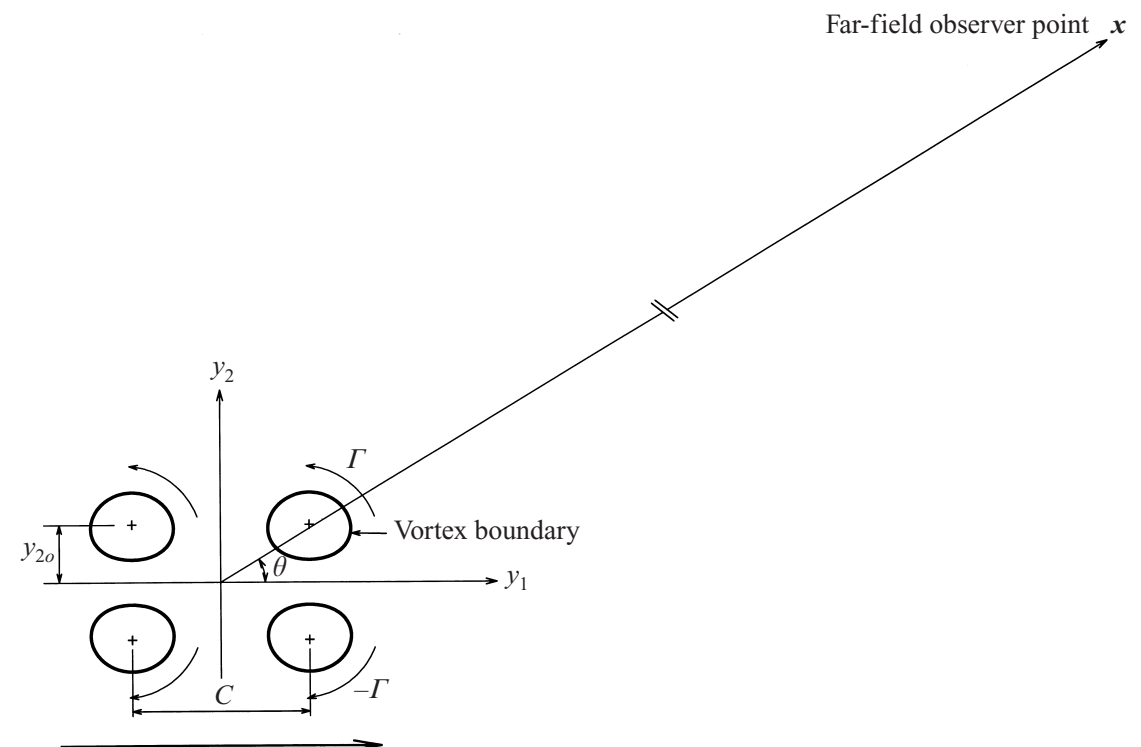

Direction of vortex pair propagation

FIGURE 1. Schematic diagram of the initial vortex pair system. +, Vortex centroid; spanwise $y_{3}$-direction is normal out of paper.

core boundaries. The streamfunction $\Psi$ in two dimensions at a point $\boldsymbol{y}_{0}$ in the flow field due to a vortex is

$$
\Psi\left(\boldsymbol{y}_{0}\right)=-\frac{1}{2 \pi} \iint \omega \ln \left|\boldsymbol{y}_{0}-\boldsymbol{y}\right| \mathrm{d} y_{1} \mathrm{~d} y_{2},
$$

where $y_{1}$ and $y_{2}$ represent the longitudinal and transverse coordinates respectively (figure 1) and the integration is done across the cross-section of the vortex cores; $\omega$ denotes vorticity at the point $\boldsymbol{y}$ inside a vortex core. The corresponding fluid velocity induced by the vortex, $\boldsymbol{u}$, is

$$
\boldsymbol{u}\left(\boldsymbol{y}_{0}\right)=\frac{1}{4 \pi} \int \omega \ln \left|\boldsymbol{y}_{0}-\boldsymbol{y}\right|^{2} \mathrm{~d} \boldsymbol{y},
$$

where $\left(\mathrm{d} \boldsymbol{y}=\mathrm{d} y_{1}, \mathrm{~d} y_{2}\right)$ and the integration is taken counter-clockwise over the vortex core boundary. If $\boldsymbol{y}_{0}$ is a point on a vortex core boundary, then (2) enables the calculation of the velocity of the core boundary. The motion of the vortex core during the interaction can thus be obtained. Full details of the method can be found in Zabusky, Hughes \& Roberts (1979) and Dritschel (1986), and thus are not repeated here. In the computations, the singularity in (2) is handled by an integration by parts (Dritschel 1986). Also, the node-point insertion and relaxation procedure employed by Pozrikidis \& Higdon (1985) were adopted so as to ensure smooth vortex core boundaries. All the contour integrals involved were computed using the four-point Gaussian quadrature procedure. The vortex core shapes were obtained by integrating the core boundary velocities with respect to time, using the fourth-order Runge-Kutta method as in Tang \& Ko (1997) and Dritschel (1986). The time step was so chosen that no visible difference of results could be found upon its further reduction.

The initial vortex core boundaries were computed using the numerical procedure of Pierrehumbert (1980). They are characterized by a variable $\alpha$, which represents the ratio of the vortex-core equivalent radius to the separation of the two vortex centroids 
of the vortex pair. The definition of the centroid of each vortex core $\boldsymbol{y}_{c}$ in the vortex pair follows that of Pierrehumbert (1980) and Tang \& Ko (1997):

$$
\boldsymbol{y}_{c}=y_{c 1} \hat{\boldsymbol{y}}_{1}+y_{c 2} \hat{\boldsymbol{y}}_{2}=\frac{\int \omega y_{1} \mathrm{~d} A}{\int \omega \mathrm{d} A} \hat{\boldsymbol{y}}_{1}+\frac{\int \omega y_{2} \mathrm{~d} A}{\int \omega \mathrm{d} A} \hat{\boldsymbol{y}}_{2} .
$$

The caret denotes unit vector. The velocity, acceleration and jerk of each vortex centroid presented in this paper were computed by differentiating $\boldsymbol{y}_{c}$ with respect to time numerically. Since the vorticity $\omega$ was taken to be constant, each vortex centroid so defined coincides with the centre of mass of the corresponding vortex core. Though Saffman (1992) has discussed the difficulty in choosing an appropriate mathematical definition for the centroid of a vorticity patch, the present definition will help in the identification of sound sources in the later stages of this paper.

Another possible definition of a centre is based on the vortex impulse, as in Pozrikidis (1986) and Tang \& Ko (1995). In the two-dimensional case, the vortex impulse $I$ is

$$
\boldsymbol{I}=\rho_{0} \int \boldsymbol{y} \times \boldsymbol{\omega} \mathrm{d} A=-\rho_{0} \hat{\boldsymbol{y}}_{1} \int y_{2} \omega \mathrm{d} A+\rho_{0} \hat{\boldsymbol{y}}_{2} \int y_{1} \omega \mathrm{d} A,
$$

where $\rho_{0}$ is the density of the ambient incompressible fluid. The impulse centre $\boldsymbol{y}_{i c}$ in the foregoing discussion is defined as

$$
\boldsymbol{y}_{i c}=y_{i c 1} \hat{\boldsymbol{y}}_{1}+y_{i c 2} \hat{\boldsymbol{y}}_{2}=\frac{\int y_{1} y_{2} \omega \mathrm{d} A}{\int \omega y_{2} \mathrm{~d} A} \hat{\boldsymbol{y}}_{1}+\frac{\int y_{1} y_{2} \omega \mathrm{d} A}{\int \omega y_{1} \mathrm{~d} A} \hat{\boldsymbol{y}}_{2} .
$$

It can be shown that $\boldsymbol{y}_{i c}=\boldsymbol{y}_{c}$ when the vortex core is symmetrical about its principle axes. Also, the condition $y_{i c 1}=y_{c 1}$ implies $y_{i c 2}=y_{c 2}$ and vice versa. The difference $\left(\boldsymbol{y}_{i c}-\boldsymbol{y}_{c}\right)$ thus relates to the degree of vortex core deformation. The above definitions of vortex core centres will be shown to be useful in the later analysis of the sound generation mechanisms. For simplicity, the common axis of the vortex pairs is chosen so as to coincide with $y_{2}=0$. The interactions in the negative- $y_{2}$ (lower) half-plane are therefore the mirror images of those occurring in the positive- $y_{2}$ (upper) half-plane.

\subsection{The sound field}

In low-Mach-number flows, the theories of Powell (1964) and Howe (1975) show that the far-field pressure $p$ generated by unsteady vortex motions can be obtained by solving the inhomogeneous wave equation

$$
\frac{1}{c^{2}} \frac{\partial^{2} p}{\partial t^{2}}-\nabla^{2} p=\rho_{0} \nabla \cdot(\boldsymbol{\omega} \times \boldsymbol{u}),
$$

where $c$ is the speed of sound in the ambient fluid. Following the method of Möhring (1978), one obtains for a point source of a small volume $\delta V$ at the near-field position $\boldsymbol{y}$ a pressure $\delta p$ at the far-field position $\boldsymbol{x}$, such that

$$
\delta p(\boldsymbol{x}, t)=\frac{\rho_{0}}{12 \pi c^{2}} \frac{\partial^{3}}{\partial t^{3}} \frac{(\hat{\boldsymbol{x}} \cdot \boldsymbol{y}) \boldsymbol{y} \cdot(\boldsymbol{\omega} \times \hat{\boldsymbol{x}})}{|\boldsymbol{x}-\boldsymbol{y}|} \delta V,
$$

where the expression being differentiated is to be evaluated at the retarded time $\tau=t-|\boldsymbol{x}-\boldsymbol{y}| / c$. Further manipulation of the above equation with the assumption of a compact vortex core gives

$$
p(\boldsymbol{x}, t)=\frac{\rho_{0}}{12 \pi c^{2}} \int \frac{1}{|\boldsymbol{x}-\boldsymbol{y}|} \frac{\partial^{3}}{\partial t^{3}}\left(\cos 2 \theta \int \omega y_{1} y_{2} \mathrm{~d} A+\sin 2 \theta \int \frac{y_{2}^{2}-y_{1}^{2}}{2} \omega \mathrm{d} A\right) \mathrm{d} y_{3},
$$


where $y_{3}$ denotes a distance in the spanwise direction and $\theta$ the observer angle, as shown in figure 1 . Without loss of generality, the far-field observer position can be chosen so that

$$
|\boldsymbol{x}-\boldsymbol{y}|=\sqrt{\left(x_{1}-y_{1}\right)^{2}+\left(x_{2}-y_{2}\right)^{2}+y_{3}^{2}},
$$

and for $x_{1} \gg y_{1}$ and $x_{2} \gg y_{2}$,

$$
\frac{1}{|\boldsymbol{x}-\boldsymbol{y}|}=\frac{1}{\left|\boldsymbol{x}^{\prime}\right|}-\frac{x_{2} y_{2}}{\left|\boldsymbol{x}^{\prime}\right|^{3}}+O\left(\left|\boldsymbol{x}^{\prime}\right|^{-3}\right)
$$

where $\left|\boldsymbol{x}^{\prime}\right|=\sqrt{\left(x_{1}-y_{1}\right)^{2}+x_{2}^{2}+y_{3}^{2}} \approx \sqrt{x_{1}^{2}+x_{2}^{2}+y_{3}^{2}}$. Since the sense of rotation of the vortex core fluids in the upper $y_{2}$-plane is opposite that in the lower $y_{2}$-plane, the second area integral on the right-hand side of (8) is $O\left(\left|x^{\prime}\right|^{-2}\right)$. The first area integral on the right-hand side of (8) is $O\left(\left|x^{\prime}\right|^{-1}\right)$ and thus, unlike the two-dimensional vortex case of Tang \& Ko (1997) in which two lateral quadrupoles co-exist, it is shown here that there is only one dominant two-dimensional lateral quadrupole field in the present study:

$$
p(\boldsymbol{x}, t)=\frac{\rho_{0}}{12 \pi c^{2}} \cos 2 \theta \int \frac{1}{|\boldsymbol{x}-\boldsymbol{y}|}\left(\frac{\partial^{3}}{\partial t^{3}} \int \omega y_{1} y_{2} \mathrm{~d} A\right) \mathrm{d} y_{3}+O\left(\left|\boldsymbol{x}^{\prime}\right|^{-2}\right) .
$$

The source of sound is, therefore, $\left(\partial^{3} / \partial t^{3}\right) \int \omega y_{1} y_{2} \mathrm{~d} A$, which is denoted by $\ddot{S}$ in the later discussions. The $y_{3}$ integration can be transformed into a time integration in the final computation of the sound field as shown in Ffowcs Williams \& Hawkings (1968) and more explicitly in Tang \& Ko (1997). However, this integration has no bearing on the sound generation mechanism, and thus was not performed in the present investigation.

\subsection{Sound generation mechanisms}

Owing to the mirror image nature of the vortex cores in the upper and lower $y_{2}$-plane, the contributions of the vortex cores in the lower $y_{2}$-plane in (10) are the same as those in the upper $y_{2}$-plane. Thus, the following discussions will be focused on the activities occurring in the upper $y_{2}$-plane.

Powell (1995) reiterated that vortex sound is generated by the accelerating rates of change of the vortex strength, as viewed from the far-field point. Any mean motion of the vortices is not expected to be sound producing, thus any mean motion involved in the source term derived in (10) must be eliminated. In the present study, the two vortex pairs are translating towards the positive longitudinal $\left(y_{1}\right)$ direction. Suppose the whole system is translating at an average speed $U$. Let $y_{1}^{\prime}=y_{1}-\left(U t+y_{1 o}\right)$, where $y_{10}$ denotes the initial longitudinal coordinate of the two vortex pair system and the prime denotes a quantity relative to the steadily translating centre of the whole system, then one obtains for a particular vortex pair that

$$
\begin{aligned}
\frac{\partial^{3}}{\partial t^{3}} \int \omega y_{1} y_{2} \mathrm{~d} A & =\frac{\partial^{3}}{\partial t^{3}} \int \omega y_{1}^{\prime} y_{2} \mathrm{~d} A+\frac{\partial^{3}}{\partial t^{3}} \int \omega\left(U t+y_{1 o}\right) y_{2} \mathrm{~d} A \\
& =\frac{\partial^{3}}{\partial t^{3}} \int \omega y_{1}^{\prime} y_{2} \mathrm{~d} A+\left(U t+y_{1 o}\right) \frac{\partial^{3}}{\partial t^{3}} \int \omega y_{2} \mathrm{~d} A+3 U \frac{\partial^{2}}{\partial t^{2}} \int \omega y_{2} \mathrm{~d} A .
\end{aligned}
$$

The integration is taken over the cross-section of the vortex pair concerned. The last two terms on the right-hand side of (11) will be cancelled by similar terms of the other vortex pair, due to the conservation of the longitudinal vortex impulse. The 
mean translating motion of the vortex system, therefore, has no effect on the sound generated. The final source term with $y_{1}^{\prime}$ does not depend on the steadily translating coordinate frame and thus can represent the actual contribution of a particular vortex pair in the sound generation process.

In order to relate the sound generation mechanisms to the dynamic parameters of the vortex pair and facilitate comparison with the results obtained from computations of circular-core vortex sound (Kambe \& Minota 1981), it is worthwhile relating the source terms in (11) to the vortex centroid and/or the impulse centre. Considering one particular vortex core, further decomposition of the source term gives

$$
\begin{aligned}
\int \omega y_{1}^{\prime} y_{2} \mathrm{~d} A & =\int \omega\left(y_{1}^{\prime}-y_{c 1}^{\prime}\right) y_{2} \mathrm{~d} A+y_{c 1}^{\prime} \int \omega y_{2} \mathrm{~d} A \\
& =\int \omega\left(y_{1}-y_{c 1}\right) y_{2} \mathrm{~d} A+\Gamma y_{c 1}^{\prime} y_{c 2} \\
& =\Gamma\left(y_{i c 1}-y_{c 1}\right) y_{c 2}+\Gamma y_{c 1}^{\prime} y_{c 2} \\
& =S_{1}+S_{2},
\end{aligned}
$$

where $S_{1}=\Gamma\left(y_{i c 1}-y_{c 1}\right) y_{c 2}, S_{2}=\Gamma y_{c 1}^{\prime} y_{c 2}$ and $\Gamma\left(=\int \omega \mathrm{d} A\right)$ is the circulation. The sound is thus generated by the third time derivatives of the two components on the right-hand side of (12). The first component $S_{1}$ clearly represents the effect of core shape or deformation as discussed before. The second component $S_{2}$ gives the part of the sound produced when the whole vortex impulse in the longitudinal direction is assumed to be carried at the vortex centroid. It relates directly to the results obtained from the circular-core assumption (Kambe \& Minota 1981; Tang \& Ko 1993), because the first component $S_{1}$ vanishes when the core is circular. These source components, $S_{1}$ and $S_{2}$, can be further decomposed as in Tang \& Ko (1995). The third time derivative of $S_{2}$, can be expressed in terms of vortex centroid motion as

$$
\ddot{S}_{2}=\frac{\partial^{3} S_{2}}{\partial t^{3}}=\Gamma\left(y_{c 2} \frac{\partial^{3} y_{c 1}^{\prime}}{\partial t^{3}}+3 \frac{\partial y_{c 2}}{\partial t} \frac{\partial^{2} y_{c 1}^{\prime}}{\partial t^{2}}+3 \frac{\partial^{2} y_{c 2}}{\partial t^{2}} \frac{\partial y_{c 1}^{\prime}}{\partial t}+y_{c 1}^{\prime} \frac{\partial^{3} y_{c 2}}{\partial t^{3}}\right) .
$$

Since $S_{2}$ does not depend on the coordinate frame, all the four terms on the right-hand side of (13) are Galilean invariants. The third time derivative of $S_{1}$ can be decomposed in the same manner. The contributions from each vortex pair can then be examined. It will be shown later that the relative importance of these four decomposed source terms depends on the type of vortex pair interaction. The physical sound generation mechanism represented by these decomposed terms will also be discussed later.

\section{Results and discussion}

In real jets, the vortical structures roll up within a relatively short distance from the nozzle exit. They then undergo leapfrogging motions and, subsequently, two structures may coalesce to form one single structure, or part of the fluid from one structure is entrained into the other (Hussain \& Clark 1981). Thus, the types of vortex interactions investigated in the present study are the leapfrogging motion, partial coalescence and coalescence.

As discussed before, the core shape of a steadily translating vortex pair is characterized by a parameter $\alpha$ (Pierrehumbert 1980). Without loss of generality, $\alpha$ was taken to be 0.5 in the present investigation. For the same type of vortex pair interaction, the results for other $\alpha$ are similar. The suffices $L$ and $T$ hereinafter denote quantities associated with the initially leading and trailing vortex pairs respectively. All the 

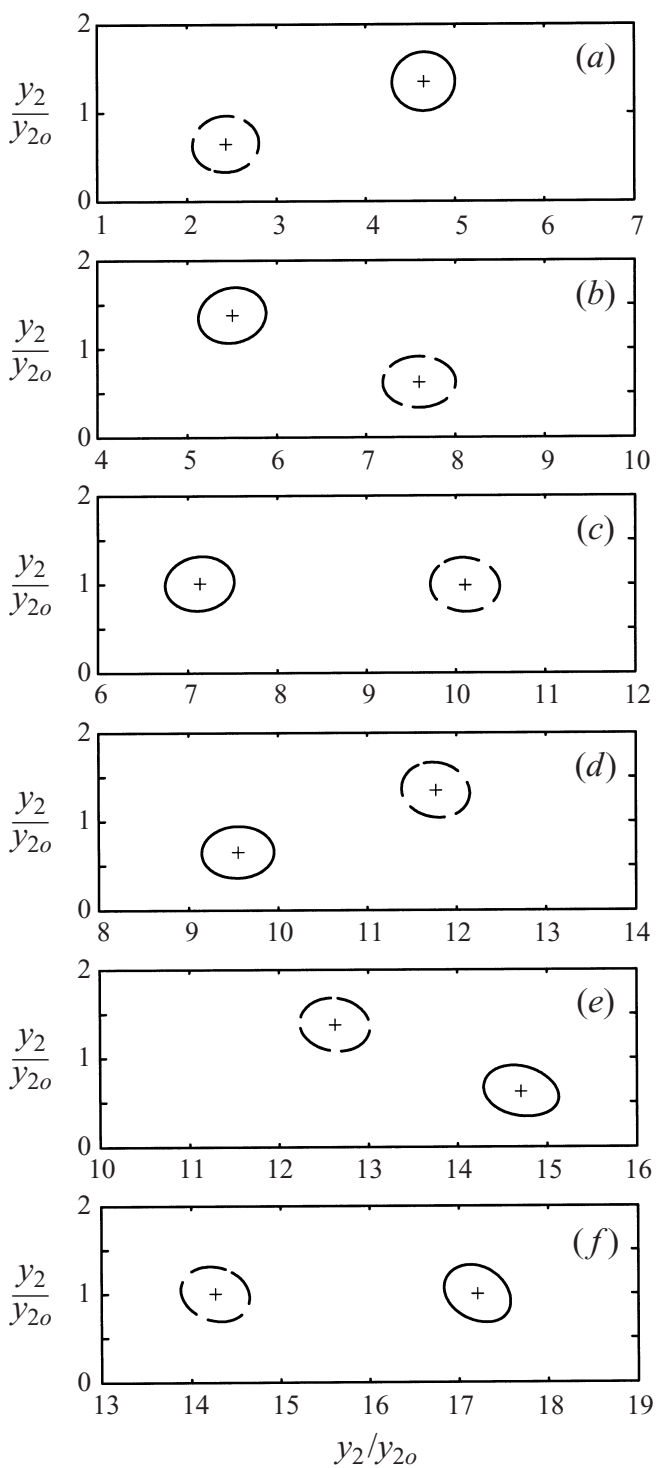

FiguRE 2. The vortex dynamics of leapfrogging motions. $C / y_{20}=3$. (a) $\omega t=50 ;(b) 100 ;(c) 150$; (d) $200 ;(e) 250 ;(f) 300$. — - Initially leading vortex pair; —— - initially trailing vortex pair; + , vortex centroid.

quantities, unless otherwise stated, are non-dimensionalized. Lengths are normalized by $y_{2 o}$ and time by $\omega^{-1}$. The velocities, accelerations and jerks are normalized by $\omega y_{2 o}, \omega^{2} y_{2 o}$ and $\omega^{3} y_{2 o}$ respectively. The source terms, such as $\ddot{S}$, are normalized by $\omega^{4} y_{20}^{4}$.

\subsection{Leapfrogging motion}

A leapfrogging interaction, similar to the mutual slip through of vortex rings (Yamada \& Matsui 1979), occurs when the strengths of the vortex pairs and their separation are of appropriate combinations (figure 2). Only the results obtained with $C / y_{2 o}=3$, where $C$ and $y_{2 o}$ are the initial vortex centroid separation and the initial transverse 

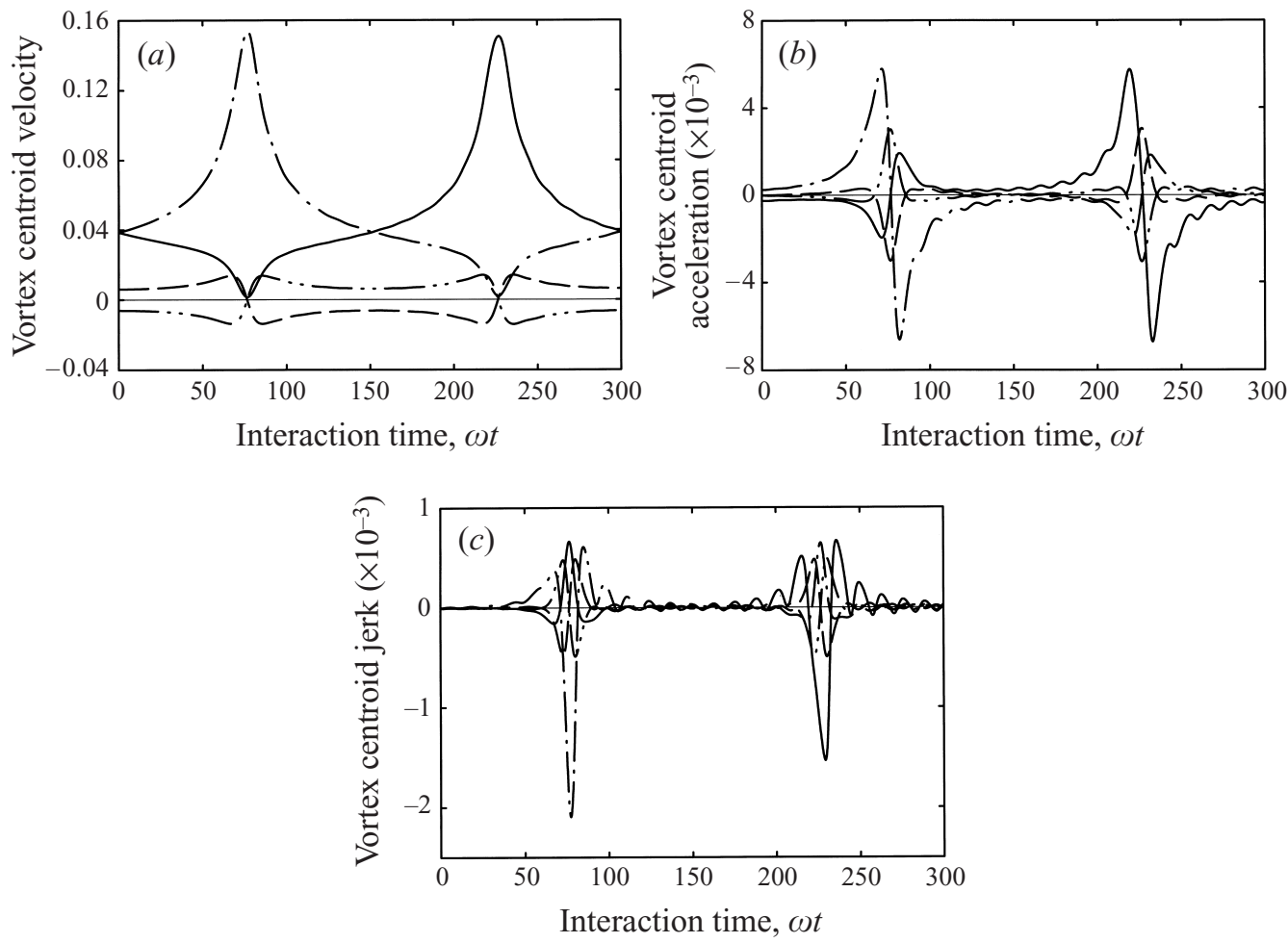

FIGURE 3. (a) Time variation of vortex centroid velocities; $(b)$ time variation of vortex centroid accelerations; $(c)$ time variation of vortex centroid jerks. $C / y_{2 o}=3 .-$, Longitudinal component of initially leading vortex pair; - —, transverse component of initially leading vortex pair; - $\cdot$ - , longitudinal component of initially trailing vortex pair; - -.- transverse component of initially trailing vortex pair.

coordinate of the vortex system respectively (figure 1), will be presented, as those for other $C / y_{20}$ are similar unless the type of interaction is different.

Our results for time variations of the vortex centroid velocities, accelerations and jerks, as shown in figure 3, are similar to those of the interaction between two circularcore vortex rings (Tang \& Ko 1993 and Kambe \& Minota 1981), except that ripples appear on the present acceleration and jerk results (figures $3 b$ and $3 c$ ). Tang \& Ko $(1995,1997)$ ascribed the appearance of such ripples to the deformation and nutation of the vortex cores during their leapfrogging motions.

Since the focus of the present study is on the source terms, their time variations, instead of the far-field pressure fluctuations, will be presented. The time variations of $\ddot{S}$ consist of a nearly sinusoidal component, which varies in amplitude after each slip-through instant at $\omega t=76.7$ and 226.8 (figure 4). The contributions from the initially leading and trailing vortex pairs, $\ddot{S}_{L}$ and $\ddot{S}_{T}$ respectively, as calculated from (11), are also included. There is clear evidence that the vortex pair having larger $y_{c 2}$ at the slip-through instant radiates higher sound power at or close to this instant when the magnitude of $\ddot{S}_{2}$ is considerably higher than those of the sinusoidal components (figures $5 a$ and $5 b$ ). Figure $5(c)$ shows the time variation of $\sum \ddot{S}_{2}\left(=\ddot{S}_{2 L}+\ddot{S}_{2 T}\right.$ ), which resembles those of the mutual threading of the circular-core vortex rings of Kambe \& Minota (1981) and Tang \& Ko (1993). Thus, this term represents the sound generation due purely to the dynamics of the vortex centroids. The core shape variation does not 


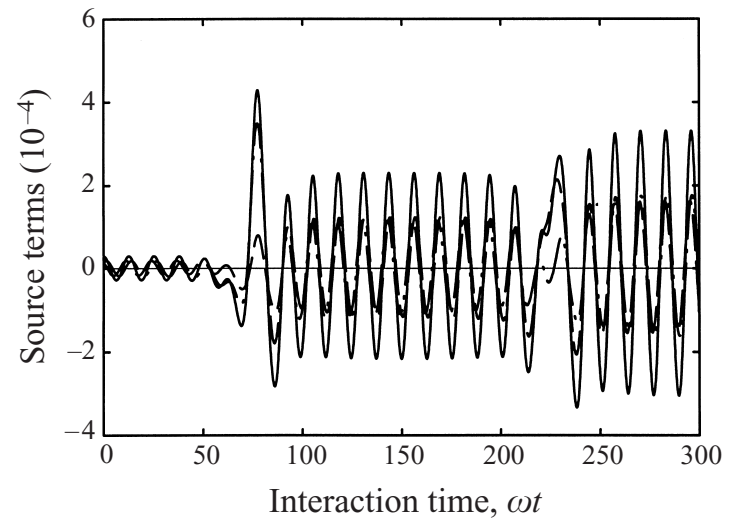

FIGURE 4. Time variation of source terms. $C / y_{2 o}=3 .-, \sum \ddot{S} ;-\cdot-, \ddot{S}_{L} ;-\longrightarrow, \ddot{S}_{T}$.
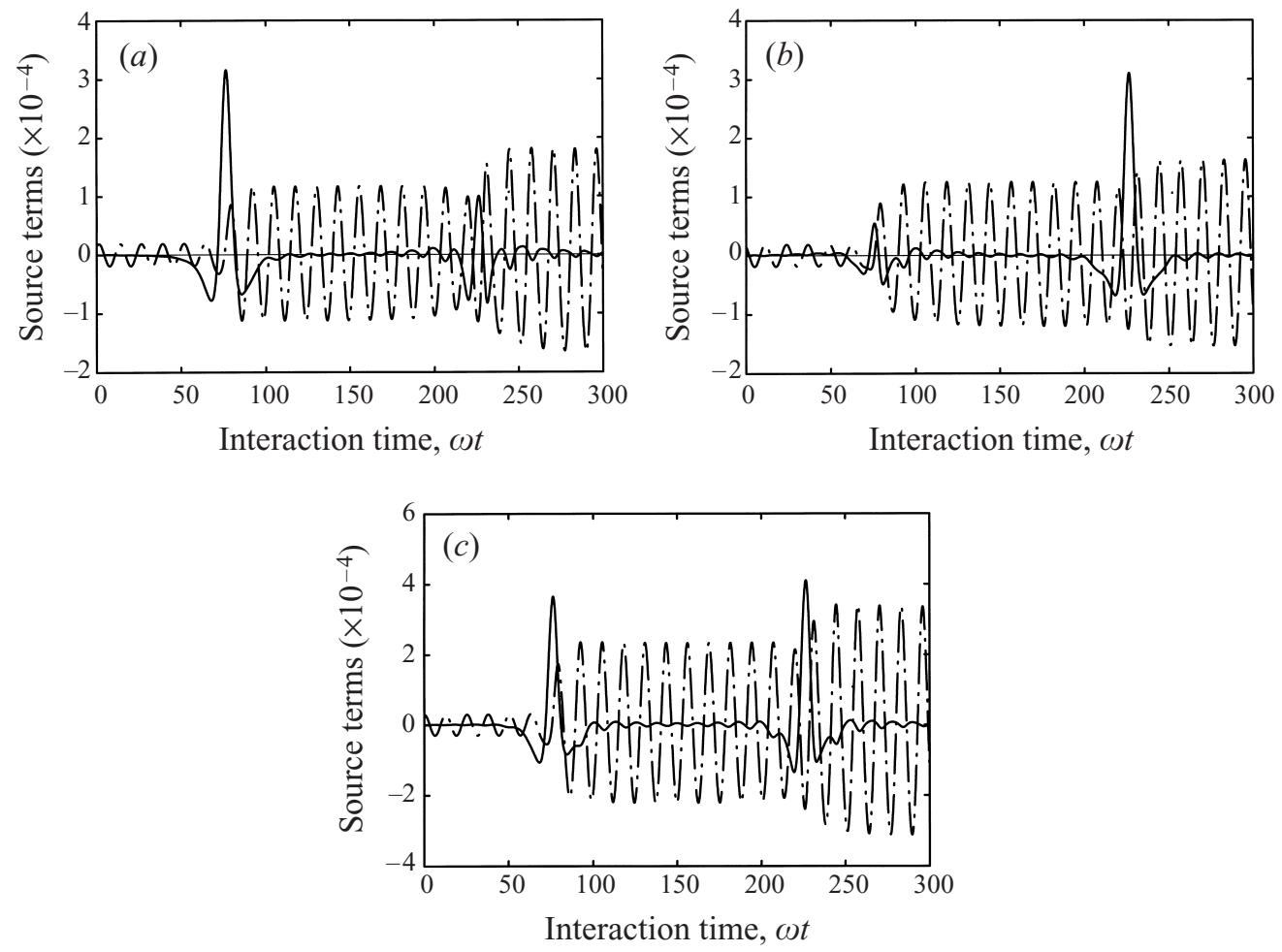

FIGURE 5. Time variation of individual vortex pair contributions in overall sound generation. $C / y_{2 o}=3(a)$ Initially leading vortex pair; (b) initially trailing vortex pair; (c) $\sum \ddot{S}_{1}$ and $\sum \ddot{S}_{2}$. $-\cdot-\ddot{S}_{1} ;-, \ddot{S}_{2}$.

have significant influence on the variation of $\sum \ddot{S}_{2}$. However, $\ddot{S}_{2}$ is only important at or close to the slip-through instants in the leapfrogging interaction (figures $5 a$ and $5 b$ ). The nearly sinusoidal fluctuations are produced by both vortex pairs. Figures $5(a)$ and $5(b)$ also confirm that the nearly sinusoidal fluctuations are related to the first source term $\ddot{S}_{1}$ and are significant after the first slip-through instant.

Decomposition of $\ddot{S}_{2}$ shows that the majority of the sound radiated by the vortex 

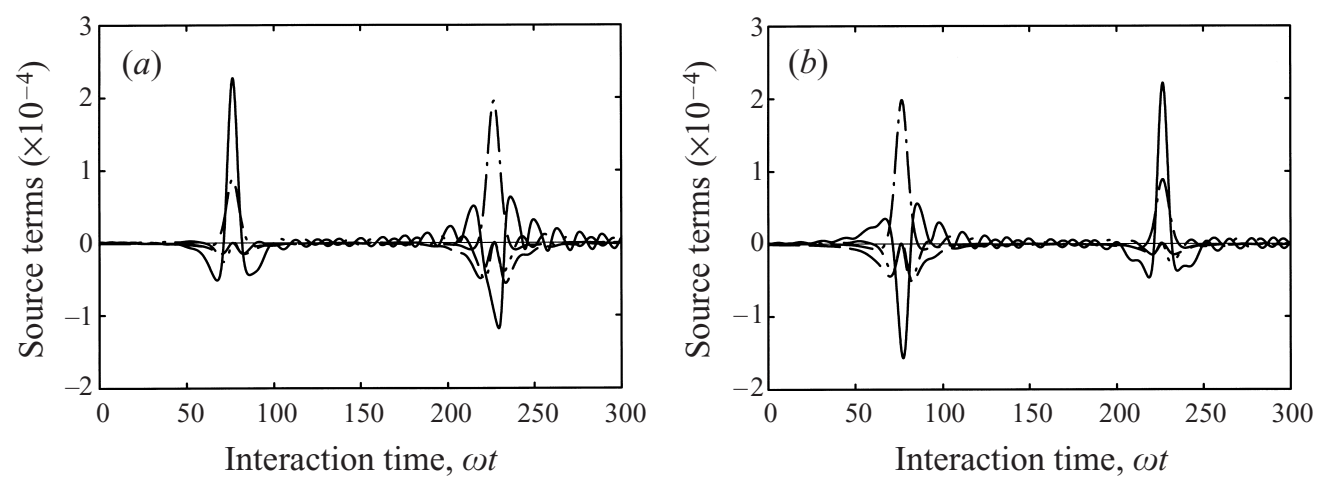

FIGURE 6. Decomposition of source term $\ddot{S}_{2}$. (a) Initially leading vortex pair; $(b)$ initially trailing vortex pair.,$- \Gamma y_{c 2} \partial^{3} y_{c 1}^{\prime} / \partial t^{3} ;-—, 3 \Gamma\left(\partial y_{c 2} / \partial t\right) \partial^{2} y_{c 1}^{\prime} / \partial t^{2} ;-\cdot-, 3 \Gamma\left(\partial^{2} y_{c 2} / \partial t^{2}\right) \partial y_{c 1}^{\prime} / \partial t ;-\cdot \cdot-$, $\Gamma y_{c 1}^{\prime}\left(\partial^{3} y_{c 2} / \partial t^{3}\right)$

centroid dynamics is related to the terms $\Gamma y_{c 2}\left(\partial^{3} y_{c 1}^{\prime} / \partial t^{3}\right)$ and $3 \Gamma\left(\partial^{2} y_{c 2} / \partial t^{2}\right) \partial y_{c 1}^{\prime} / \partial t$, the first and the third decomposed terms of (13) respectively (figures $6 a$ and $6 b$ ). Though $y_{c 1}^{\prime}$ denotes a distance relative to a steadily moving frame, its third time derivative is the jerk of a vortex centroid. The term $\Gamma y_{c 2}\left(\partial^{3} y_{c 1}^{\prime} / \partial t^{3}\right)$ is related to this jerk and the vortex impulse carried by each vortex pair. Thus, relatively higher sound power is produced during the large longitudinal jerking motions of the vortex centroids.

The term $3 \Gamma\left(\partial^{2} y_{c 2} / \partial t^{2}\right) \partial y_{c 1}^{\prime} / \partial t$ requires deeper thought. It represents the coupling between the transverse acceleration and the longitudinal velocity of a vortex centroid. Though the two time derivatives represent the rate of change of some relative distance and velocity in the near field, they can hardly be related to the vector area of Powell (1995) or the Coriolis acceleration suggested by Doak (1998). It can be observed from figure $3(b)$ that the magnitudes of the transverse acceleration of each vortex centroid close to the slip-through instants are roughly the same, while those of the longitudinal velocities and this source term vary (figures $3 a$ and 6). It is believed that the velocity $\partial y_{c 1}^{\prime} / \partial t$ is more important in the sound generation process. Since $\Gamma$ is a constant, it follows that $\Gamma\left(\partial y_{c 1}^{\prime} / \partial t\right)$ represents the total vortex force $F_{y 2}$ acting on a vortex core of a vortex pair in the transverse direction (Saffman 1992). The term $3 \Gamma\left(\partial^{2} y_{c 2} / \partial t^{2}\right) \partial y_{c 1}^{\prime} / \partial t$ is related to this vortex force $F_{y 2}$ and the transverse accelerating motion of a vortex centroid. Combining the contributions from the two vortex pairs, one obtains

$$
\sum \Gamma \frac{\partial y_{c 1}^{\prime}}{\partial t} \frac{\partial^{2} y_{c 2}}{\partial t^{2}}=\frac{\partial^{2} y_{c 2, L}}{\partial t^{2}} \Gamma\left(\frac{\partial y_{c 1, L}^{\prime}}{\partial t}-\frac{\partial y_{c 1, T}^{\prime}}{\partial t}\right),
$$

as the transverse accelarations of the vortex centroids have the same magnitudes but are $180^{\circ}$ out of phase. The resultant sound radiation represented by this source term (equation (14)) is therefore actuated by an imbalance in the transverse vortex force on the cores of the vortex pairs when the two vortex centroids are accelerating. The magnitude of the sound so generated is highest at the slip-through instant when the two vortex centroids are accelerating towards each other on the same vertical plane.

The component $S_{1}$ (equation (12)) represents the source due to the relative separation between the vortex centroid and the impulse centre of a vortex core. It is a quantity describing the non-symmetrical distribution of vortex impulse relative to the centre of mass of the core fluid, and thus depends significantly on the shape of the vortex core. $\ddot{S}_{1}$ can be decomposed in the same way as depicted in (13). Figure 7 


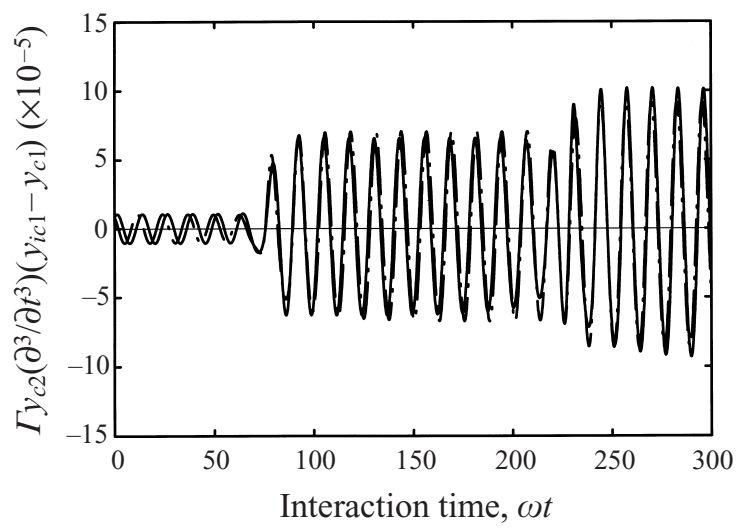

FIGURE 7. Time variation of $\Gamma y_{c 2}\left(\partial^{3} / \partial t^{3}\right)\left(y_{i c 1}-y_{c 1}\right)$ in $\ddot{S}_{1} . C / y_{2 o}=3$. - Initially leading vortex pair; - - - initially trailing vortex pair.

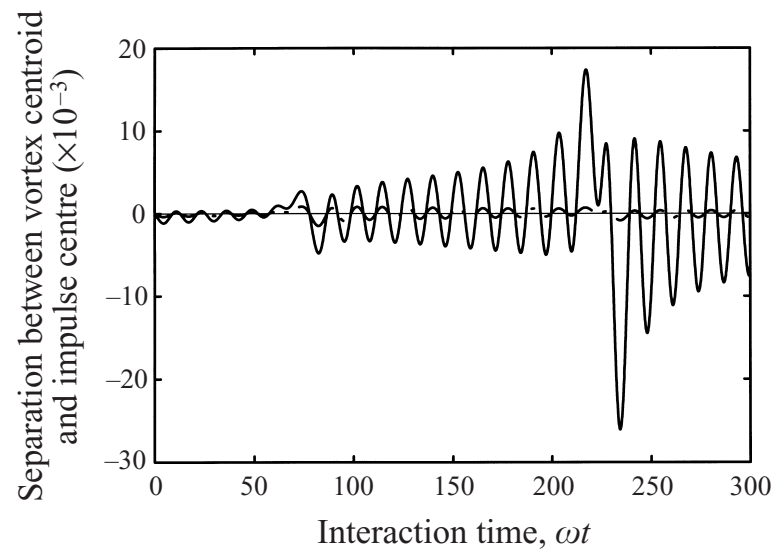

FIGURE 8. Time variation of spatial separation between vortex centroid and impulse centre for initially leading vortex pair. $C / y_{2 o}=3$.,$-\left(y_{i c 1}-y_{c 1}\right) ;-\cdot-,\left(y_{i c 2}-y_{c 2}\right)$.

suggests that the sinusoidal fluctuations on $\ddot{S}_{1}$ are due solely to $\Gamma y_{c 2}\left(\partial^{3} / \partial t^{3}\right)\left(y_{i c 1}-y_{c 1}\right)$. Therefore, the remaining terms in the decomposition will not be discussed.

The difference $\left(y_{i c 1}-y_{c 1}\right)$ is actually very small when compared to the core size (figure 8). The difference $\left(y_{i c 2}-y_{c 2}\right)$ is even smaller. The third-order time differentiation of the former amplifies its higher frequency components and thus its significance in the sound generation process. It is obvious that the larger the difference between $y_{i c 1}$ and $y_{c 1}$, the greater the asymmetry of vortex impulse distribution and the more serious the core deformation. Taking the initial leading vortex pair as an example and focusing on the instants of large $\left|y_{i c 1}-y_{c 1}\right|($ at $\omega t=133.4$ and 139.8) and the instants of $y_{i c 1}=y_{c 1}$ (at $\omega t=130.3$ and 136.4) (figure 8), the largest separation of the two centres appears when the core is severely deformed from symmetry about the longitudinal or transverse planes (figure 9). The smallest departure from symmetry is observed when $y_{i c 1}=y_{c 1}$ (thus, $y_{i c 2}=y_{c 2}$ and the two centres coincide). A measure of the rate of change of such asymmetry is, therefore, the rate of change of $\left(y_{i c 1}-y_{c 1}\right)$ as the higher frequency components in $\left(y_{i c 1}-y_{c 1}\right)$ and $\left(y_{i c 2}-y_{c 2}\right)$ are in-phase (figure 8). Since these frequency components are nearly sinusoidal, one expects that $(\partial / \partial t)\left(y_{i c 1}-y_{c 1}\right)$ is, to a large extent, proportional to $\left(\partial^{3} / \partial t^{3}\right)\left(y_{i c 1}-y_{c 1}\right)$, and is thus highly correlated with the 


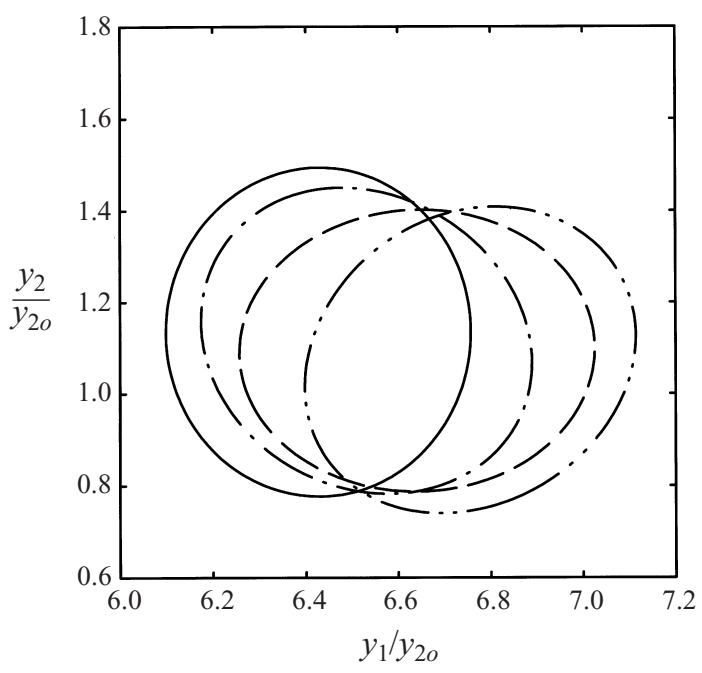

Figure 9. Shape of initially leading vortex-pair core. $C / y_{2 o}=3 .-, \omega t=130.3 ;-\cdot-, 133.4$; - - $136.4 ;-\cdots-, 139.8$.

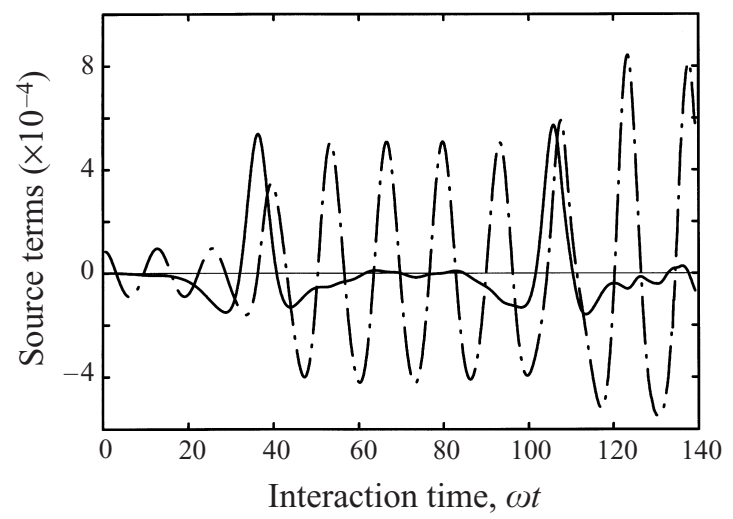

FIGURE 10. Time variation of source terms. $C / y_{2 o}=2 .-\cdot-, \sum \dddot{S}_{1} ;-, \sum \dddot{S}_{2}$.

source term $\Gamma y_{c 2}\left(\partial^{3} / \partial t^{3}\right)\left(y_{i c 1}-y_{c 1}\right)$. A high rate of change of $\left(y_{i c 1}-y_{c 1}\right)$ appears when the vortex centroid coincides with the impulse centre, that is, at the instant when the vortex core is symmetrical about the longitudinal and transverse planes. The same is also true for the initially trailing vortex pair (not shown here). Therefore, it is evident that the variation of core shape, which results in uneven distribution of vortex impulse about the vortex centroid, is a significant source of sound. This variation in the vortex core is referred to as the microscopic vortex core dynamics in the present study.

Figure 8 also shows that the rate of change of $\left(y_{i c 1}-y_{c 1}\right)$ is relatively higher at the slip-through instant. This explains the increase in the magnitude of the sinusoidal fluctuations in $\ddot{S}_{1}$ after these instants (figure 5). The frequency of these fluctuations does not depend on $C / y_{20}$, but on $\alpha$ (not shown here). The nearly constant amplitude of these fluctuations between successive slip-through instants suggests the increase and decrease of the magnitude of the corresponding core asymmetry, when a vortex core moves towards and away from the common axis $\left(y_{2}=0\right)$ respectively. Higher sound power is radiated at the instants of large rate of change of vortex core asymmetry. 

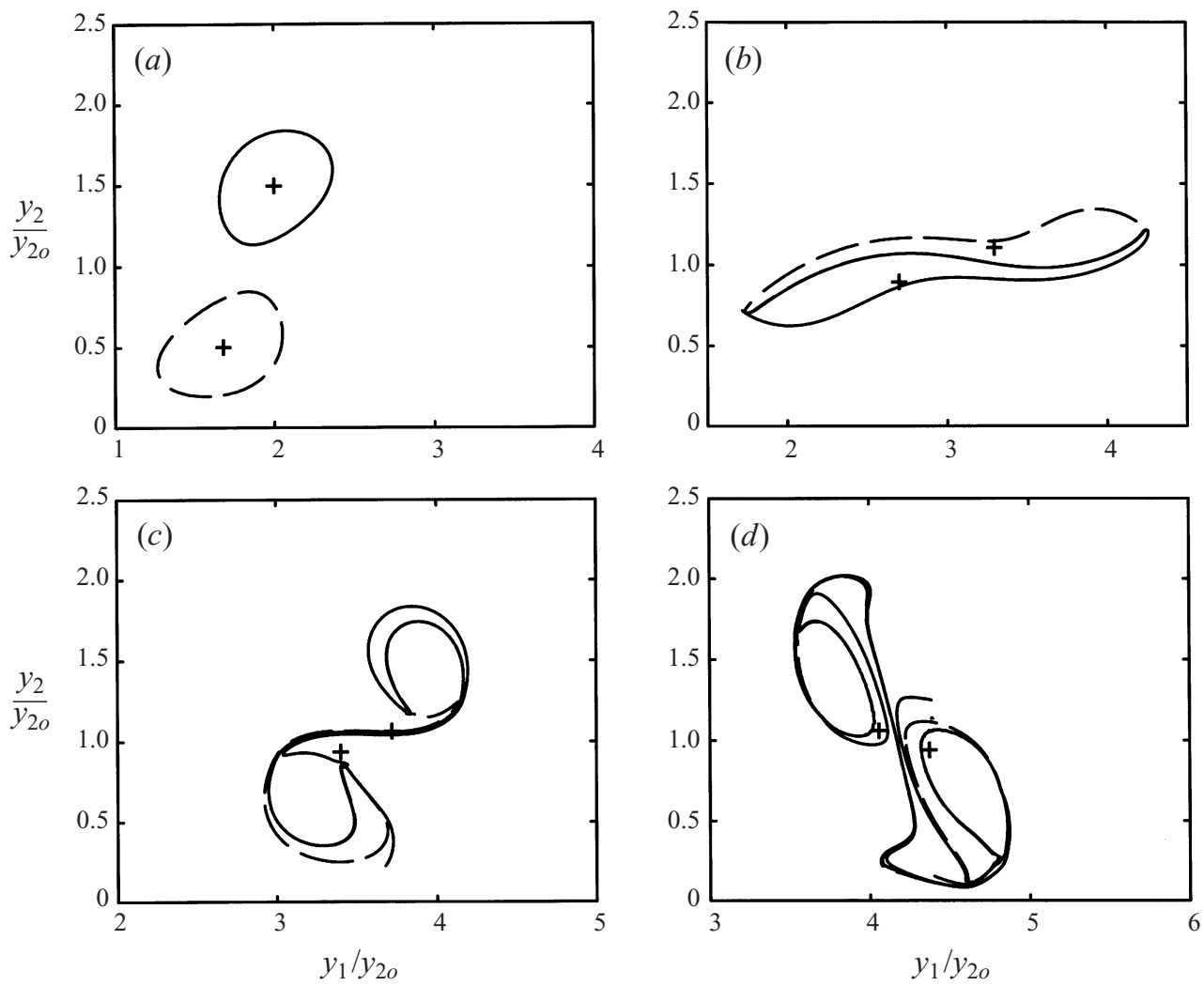

Figure 11. Vortex dynamics of partial coalescence. $C / y_{2 o}=1.5$. (a) $\omega t=20 ;(b) 40 ;(c) 50 ;(d) 60$. - Initially leading vortex pair; _ _ - initially trailing vortex pair; + , vortex centroid.

A decrease in $C / y_{20}$ shortens the interval between successive slip-through instants, resulting in larger vortex core deformation. The sound field is, therefore, dominated by the nearly sinusoidal fluctuations generated by the microscopic vortex core dynamics. The results at $C / y_{2 o}=2$ are illustrated in figure 10 .

The results presented in this section show that the sound generated during the leapfrogging motion of two identical inviscid vortex pairs is related to the longitudinal jerking motion, the unbalanced transverse vortex force when the vortex centroids are under high transverse acceleration, and the high rate of change of the vortex core asymmetry. The last item becomes more dominant when the initial vortex centroid separation decreases.

\subsection{Partial coalescence}

As the separated vortex cores in an inviscid medium cannot come into contact, coalescence is defined here as the mutual 'folding together' of the vortex cores, as described by Jacobs \& Pullin (1985).

Partial coalescence between the vortex pairs is found at $C / y_{2 o}=1.5$ (figure 11). It can be observed that large elongation of both vortex cores occurs after the first slip-through instant (figure 11b). Part of the fluid of one vortex core of one vortex pair rolls up with some of the other vortex pair, forming two patches of vorticity, each of which contains fluids from the two initial vortex cores (figures $11 \mathrm{c}$ and 11d).

The dynamics of the vortex centroids at $C / y_{2 o}=1.5$ differ substantially from those 


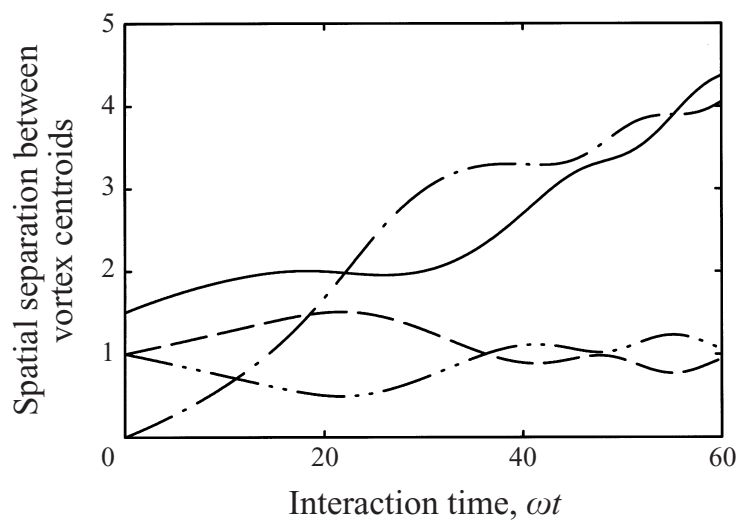

FiguRE 12. Path of individual vortex centroid during partial coalescence. $C / y_{2 o}=1.5$. $-y_{c 1}$ of initially leading vortex pair; - _, $y_{c 2}$ of initially leading vortex pair; - . - , $y_{c 1}$ of initially trailing vortex pair; - - -,$y_{c 2}$ of initially trailing vortex pair.
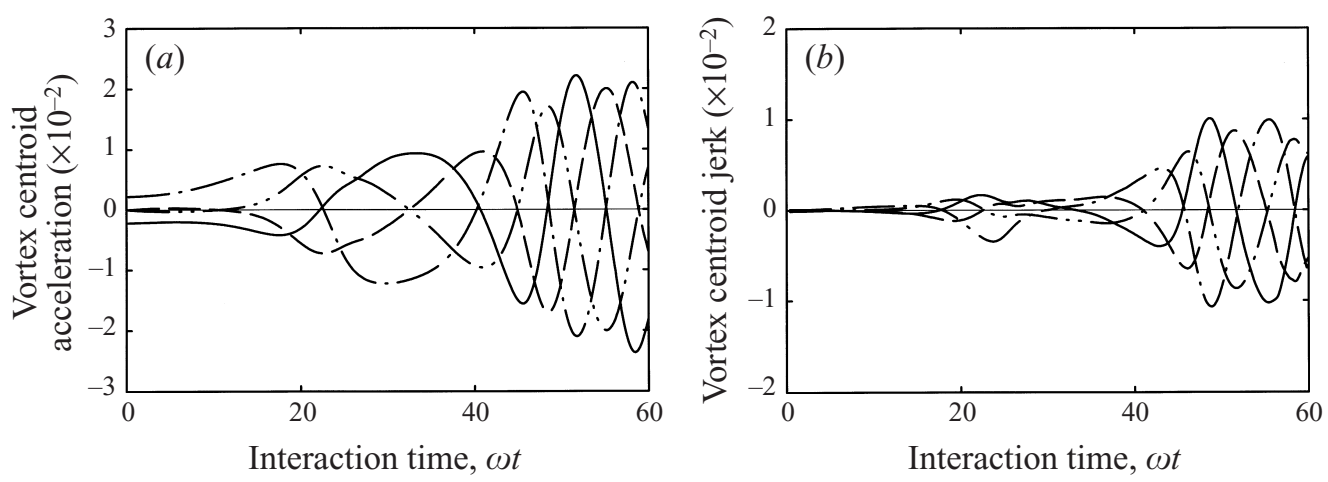

FIGURE 13. Time variation of vortex centroid dynamic parameters during partial coalescence. $C / y_{2 o}=1.5$. (a) Accelerations; $(b)$ jerks. - , Longitudinal component of initially leading vortex pair; _ - , transverse component of initially leading vortex pair; - . - , longitudinal component of initially trailing vortex pair; - - - - transverse component of initially trailing vortex pair.

in the leapfrogging case. The vortex centroid of the initially trailing vortex core is always further away from the common axis than that of the initially leading one after the slip through at $\omega t=22$ (figure 12). It seems that the structure formed by the partial coalescence process tends to hinder the rotation of the whole system (figure 11d). The overall structure rocks as it propogates in the longitudinal direction.

The rocking motion of the system after the slip through at $\omega t=22$ results in relatively higher rates of change of vortex centroid accelerations and jerks in both directions (figure 13). Unlike the results obtained in the leapfrogging cases, the jerks of the vortex centroids are of nearly the same magnitude, though out of phase. Since the differences in the transverse coordinates of these centroids after the slip through at $\omega t=22$ are not significant, especially at the instants of large jerks (figure 12), the $\ddot{S}_{2}$ from the two vortex pairs tends to cancel each other. This reduces the significance of this source in the overall sound generation, though it still forms the major part of the contribution from the individual vortex pair. Similar cancellation between the decomposed source terms has been observed by Colonius et al. (1997), though their approach and the decomposed terms are completely different from those presented here. 


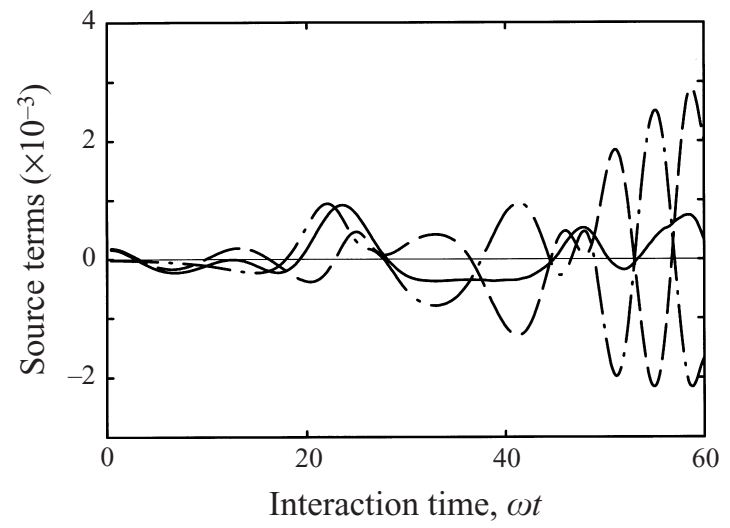

FIGURE 14. Time variation of source terms of partial coalescence. $C / y_{20}=1.5$. - , Total generation $\sum\left(\dddot{S}_{1}+\dddot{S}_{2}\right) ;-\cdot-$, initially leading vortex pair; - —, initially trailing vortex pair.

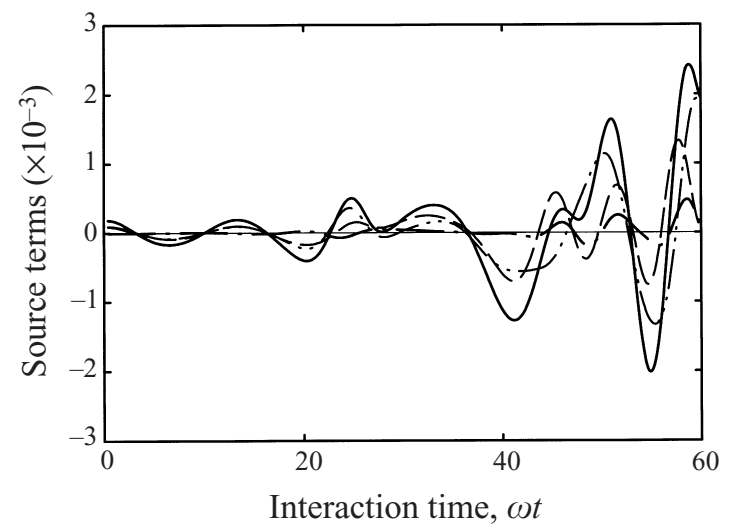

FiguRE 15. Sound generation by microscopic vortex core dynamics of partial coalescence. $C / y_{2 o}=1.5 .-, \sum \ddot{S}_{1} ;-\cdots, R ;--, \Gamma y_{c 2}\left(\partial^{3} / \partial t^{3}\right)\left(y_{i c 1}-y_{c 1}\right)$ of initially leading vortex pair; - - - $\Gamma y_{c 2}\left(\partial^{3} / \partial t^{3}\right)\left(y_{i c 1}-y_{c 1}\right)$ of initially trailing vortex pair.

Figure 14 shows the time variations of the source strengths. The first peak at or close to the slip through at $\omega t=22$ is due to the mechanism discussed in the previous section. Both $\sum \ddot{S}_{1}$ and $\sum \ddot{S}_{2}$ are important, even though they tend to counterbalance each other for $\omega t>30$, resulting in less sound generation. This phenomenon is not observed in the leapfrogging case, where these two sources appear to be independent.

Figure 15 shows the time variations of $\sum \Gamma y_{c 2}\left(\partial^{3} / \partial t^{3}\right)\left(y_{i c 1}-y_{c 1}\right)$ and the remainder $R\left(=\sum \ddot{S}_{1}-\sum \Gamma y_{c 2}\left(\partial^{3} / \partial t^{3}\right)\left(y_{i c 1}-y_{c 1}\right)\right)$. It is clear that the term $R$ is insignificant in the overall sound generation, especially at $\omega t<30$. This is due to the cancelling effect of the individual contributions. This agrees with the conclusion of Colonius et al. (1997) that the acoustic analogy may lead to terms which produce negligible acoustic field. Therefore, the sound produced by the microscopic core dynamics is, again, mainly associated with $\Gamma y_{c 2}\left(\partial^{3} / \partial t^{3}\right)\left(y_{i c 1}-y_{c 1}\right)$. The rate of change of the imbalance of vortex impulse about the vortex centroid is, therefore, closely related to the production of sound, as in the leapfrogging case. It should be noted that, since the vortex cores are severely deformed in this partial coalescence case, the relationship between the term 


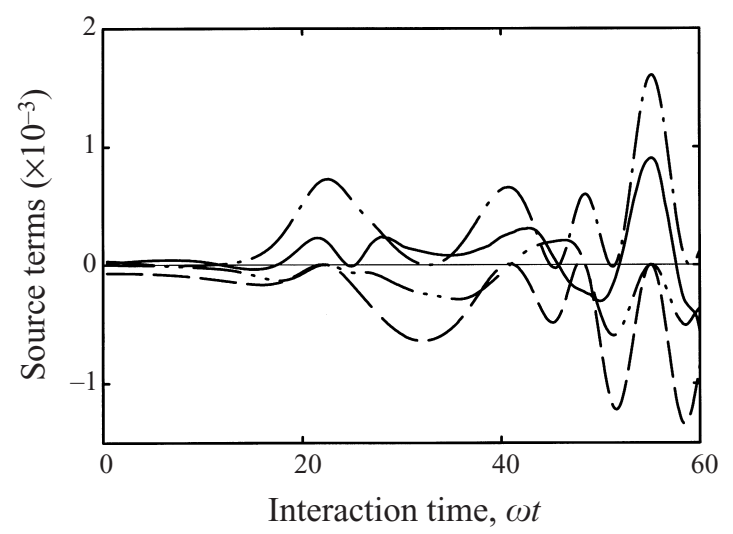

FiguRE 16. Time variation of source terms due to vortex centroid dynamics during partial coalescence. $C / y_{2 o}=1.5 .-, \sum \Gamma y_{c 2}\left(\partial^{3} y_{c 1}^{\prime} / \partial t^{3}\right) ;--, \sum 3 \Gamma\left(\partial y_{c 2} / \partial t\right) \partial^{2} y_{c 1}^{\prime} / \partial t^{2} ;-\cdot-$, $\sum 3 \Gamma\left(\partial y_{c 1}^{\prime} / \partial t\right) \partial^{2} y_{c 2} / \partial t^{2} ;-\cdots-, \sum \Gamma y_{c 1}^{\prime}\left(\partial^{3} y_{c 2} / \partial t^{3}\right)$.

$\left(\partial^{3} / \partial t^{3}\right)\left(y_{i c 1}-y_{c 1}\right)$ and the vortex core asymmetry established in the leapfrogging case is no longer valid.

The term $\sum \ddot{S}_{2}$ represents the complicated interaction of the sources, since

$$
\sum \dddot{S}_{2}=\Gamma\left(\sum y_{c 2} \frac{\partial^{3} y_{c 1}^{\prime}}{\partial t^{3}}+3 \sum \frac{\partial y_{c 2}}{\partial t} \frac{\partial^{2} y_{c 1}^{\prime}}{\partial t^{2}}+3 \sum \frac{\partial^{2} y_{c 2}}{\partial t^{2}} \frac{\partial y_{c 1}^{\prime}}{\partial t}+\sum y_{c 1}^{\prime} \frac{\partial^{3} y_{c 2}}{\partial t^{3}}\right)
$$

None of the four decomposed terms dominates the sound generation process for $\omega t>30$ (figure 16). The peaks in the time variations of $\sum \dddot{S}_{2}$ are related to the first and third decomposed terms, and the troughs to the second and fourth terms. As discussed before, the first term relates to the production of sound through the longitudinal jerking motions of the vortex pair. Since $\partial^{3} y_{c 1, L} / \partial t^{3} \approx-\partial^{3} y_{c 1, T} / \partial t^{3}$ (figure $13 b$ ), it also represents the sound production due to an imbalance of the longitudinal vortex impulse, when there is a change in the vortex pair accelerations in the same direction. The third term represents the effect of the imbalance in the transverse vortex force, while the fourth term is similar to the first term, except that the changes are in the transverse direction. The second term is more difficult to interpret. Since the relationship for the longitudinal jerks does not apply to the longitudinal accelerations (figure 13a), this term represents the sound generated when there is a relative longitudinal acceleration between the vortex pairs, which are also moving in the transverse direction. In other words, there is a non-zero vortex force in the longitudinal direction acting on each vortex core.

The relatively more important source terms in this partial coalescence interaction are the second and the third source terms, as suggested by figure 16. The sum of these two terms is

$$
3 \Gamma \frac{\partial}{\partial t} \sum\left(\frac{\partial y_{c 2}}{\partial t} \frac{\partial y_{c 1}^{\prime}}{\partial t}\right)
$$

which represents the rate of change of the total power developed by the transverse components of the vortex forces. It also represents that developed by the longitudinal components of these forces, though $\partial y_{c 1}^{\prime} / \partial t$ is not the actual velocity of a vortex centroid. It is because the effect of the steady translation velocity $U$ vanishes once the summation is considered. It should be noted that the overall power developed by each component of the vortex forces is the same. 

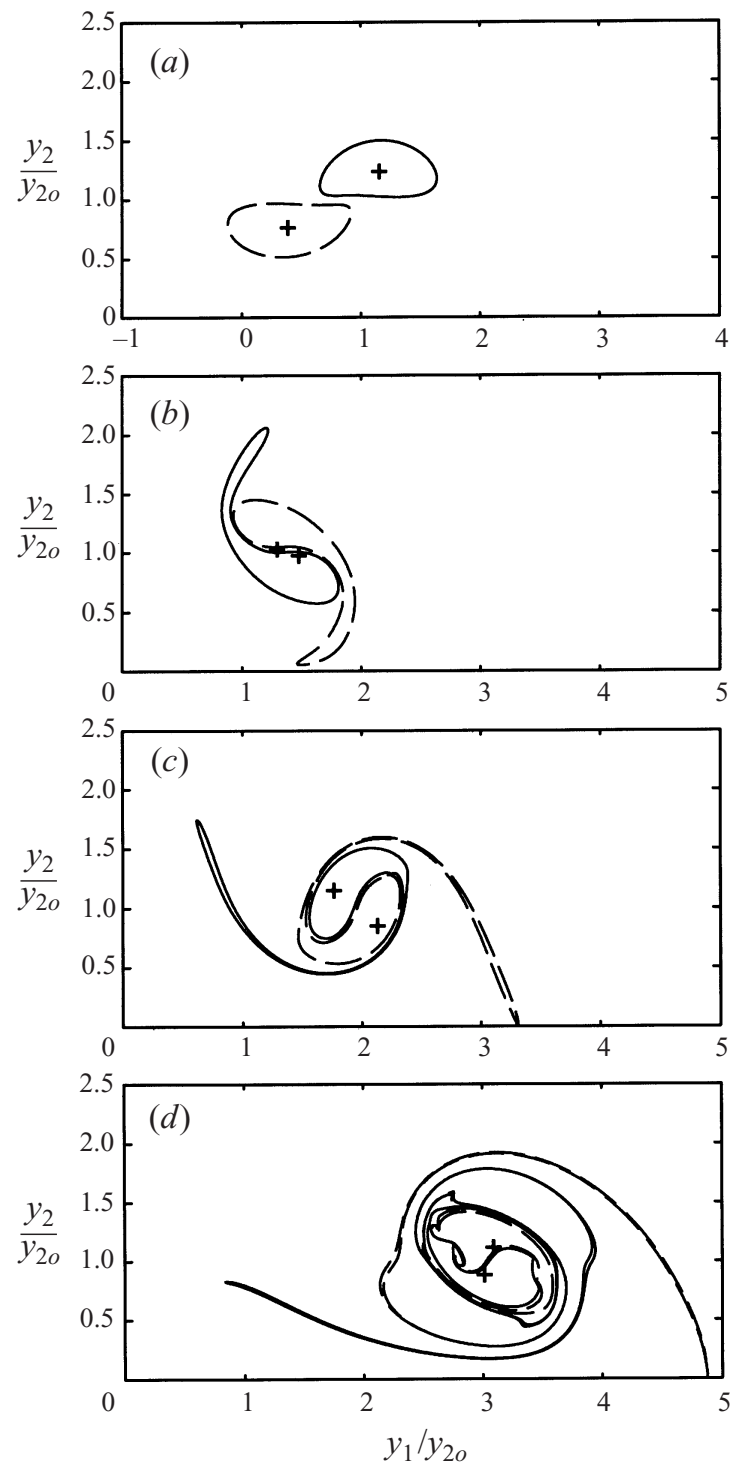

Figure 17. Vortex dynamics of coalescence. $C / y_{2 o}=1$. (a) $\omega t=5 ;(b) 15 ;(c) 25 ;(d) 45$. , Initially leading vortex pair; $-\ldots$, initially trailing vortex pair; + , vortex centroid.

\subsection{Coalescence}

Further reduction in $C / y_{2 o}$ results in severe core deformation at the initial stage of the vortex pair interaction. Coalescence, which is similar to that observed by many researchers, such as Winant \& Browand (1974), Yamada \& Matsui (1979) and Tang \& Ko (1995), occurs.

Figure 17 illustrates the coalescence at $C / y_{2 o}=1$. Strips of vorticity are observed at the front and at the back of the coalescing vortex pairs. The rapid motions of the vortex centroids and their high velocities, accelerations and jerks are expected, and thus not discussed. The main difference between these quantities and those in the leapfrogging and partial coalescence is that, apart from the relationships of the time derivatives of $y_{c 2}$ due to the conservation of vortex impulse in the longitudinal 


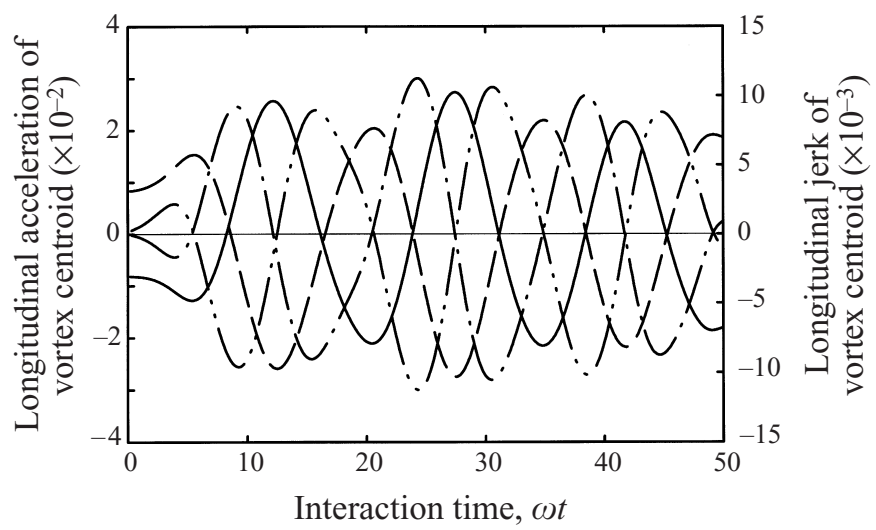

FIGURE 18. Time variation of longitudinal accelerations and jerks of vortex centroids during coalescence. $C / y_{2 o}=1$. — , Acceleration of initially leading vortex centroid; - - - , jerk of initially leading vortex centroid; _ _ , acceleration of initially trailing vortex centroid; $\_. \cdot-$, jerk of initially trailing vortex centroid.

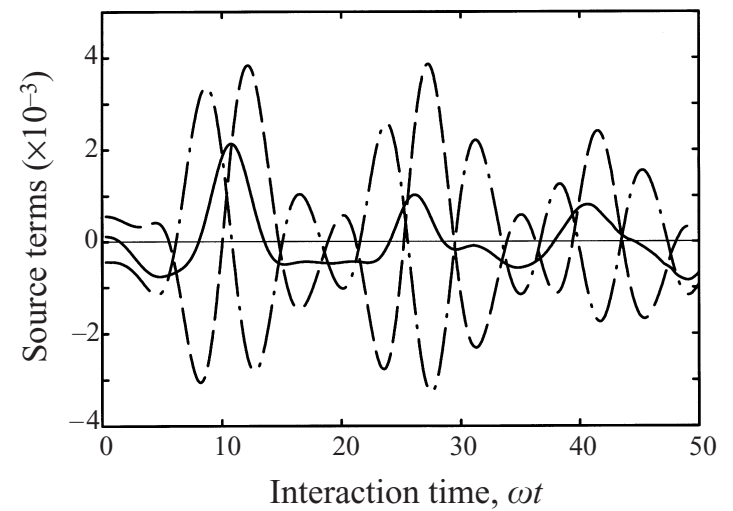

FIGURE 19. Variations of source strengths in coalescence. $C / y_{2 o}=1 .-, \sum \dddot{S} ;-\longrightarrow, \sum \dddot{S}_{1}$; - - $\sum \dddot{S}_{2}$.

direction:

one finds that during coalescence

$$
\frac{\partial^{n} y_{c 2, L}}{\partial t^{n}}=-\frac{\partial^{n} y_{c 2, T}}{\partial t^{n}}
$$

$$
\frac{\partial^{n} y_{c 1, L}^{\prime}}{\partial t^{n}} \approx-\frac{\partial^{n} y_{c 1, T}^{\prime}}{\partial t^{n}}
$$

Some typical examples of the relationship are shown in figure 18. The net vortex force in the transverse direction is, therefore, smaller than those in the leapfrogging case, though the magnitudes of the vortex centroid jerks and acceleration may be higher in this type of interaction. The sound power produced by coalescence (figure 19) is lower than that from the leapfrogging (figure $5 c$ ), though the vortex pair system in coalescence has a higher kinetic energy content. This observation is similar to that in the vortex merger studied by Mitchell, Lele \& Moin (1995).

It can also be observed from figure 19 that the contributions from $\sum \ddot{S}_{1}$ and $\sum \ddot{S}_{2}$ in the overall sound generation are equally significant. $\sum \ddot{S}_{1}$ in the coalescence is, 


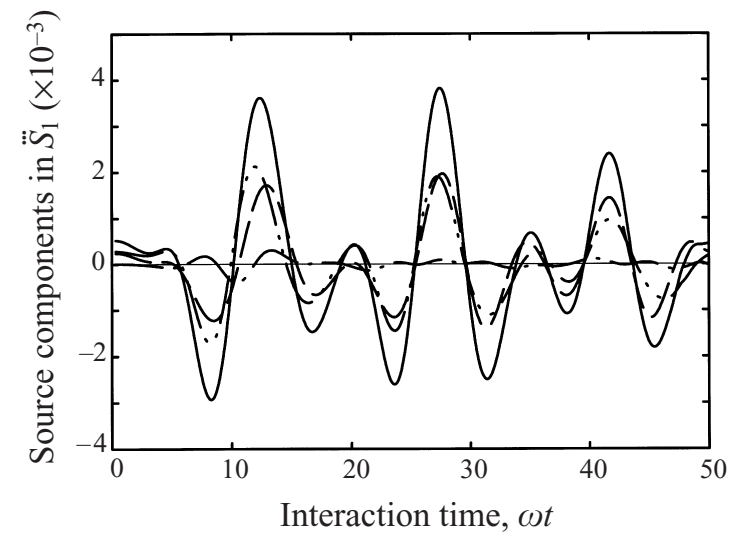

FIGURE 20. Relative importance of different components in $\sum \dddot{S}_{1}$ during coalescence. $C / y_{2 o}=1$. ,$- \sum \Gamma y_{c 2}\left(\partial^{3} / \partial t^{3}\right)\left(y_{i c 1}-y_{c 1}\right) ;--, R ;--, \Gamma y_{c 2}\left(\partial^{3} / \partial t^{3}\right)\left(y_{i c 1}-y_{c 1}\right)$ of initially leading vortex pair; - - -,$\Gamma y_{c 2}\left(\partial^{3} / \partial t^{3}\right)\left(y_{i c 1}-y_{c 1}\right)$ of initially trailing vortex pair.

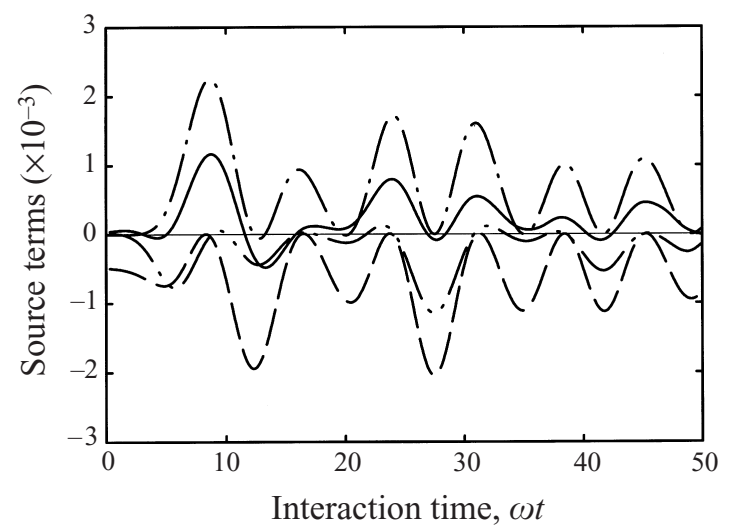

FIGURE 21. Time variations of source terms due to vortex centroid dynamics during coalescence. $C / y_{2 o}=1 .-, \sum \Gamma y_{c 2}\left(\partial^{3} y_{c 1}^{\prime} / \partial t^{3}\right) ;-—, \sum 3 \Gamma\left(\partial y_{c 2} / \partial t\right) \partial^{2} y_{c 1}^{\prime} / \partial t^{2} ;-\cdot-, \sum 3 \Gamma\left(\partial y_{c 1}^{\prime} / \partial t\right) \partial^{2} y_{c 2} / \partial t^{2}$; $-\cdot-, \sum \Gamma y_{c 1}^{\prime}\left(\partial^{3} y_{c 2} / \partial t^{3}\right)$.

again, dominated by the contribution from $\sum \Gamma y_{c 2}\left(\partial^{3} / \partial t^{3}\right)\left(y_{i c 1}-y_{c 1}\right)$. The remainder $R$ is far less important due to a similar phenomenon to that discussed in the preceding section (figure 20). Also, the contributions from both vortex pairs in this source term are of the same importance (figure 20).

In coalescence, the spatial separation between the vortex centroids is relatively small. There is a tendency for it to decrease as the flight time increases, as in the two-dimensional vortex case of Tang \& Ko (1997) (not shown here). This, together with the relationship shown in (17), suggests that the source terms $\sum \Gamma y_{c 2}\left(\partial^{3} y_{c 1}^{\prime} / \partial t^{3}\right)$ and $\sum \Gamma y_{c 1}^{\prime}\left(\partial^{3} y_{c 2} / \partial t^{3}\right)$, which depend on the relative positions of the vortex centroids or the imbalance of the vortex impulse, eventually become insignificant in the overall sound generation (figure 21). The mechanism through which sound is generated by the dynamics of the vortex centroids during the coalescence is then related to the power developed by the vortex forces, as in the partial coalescence. 

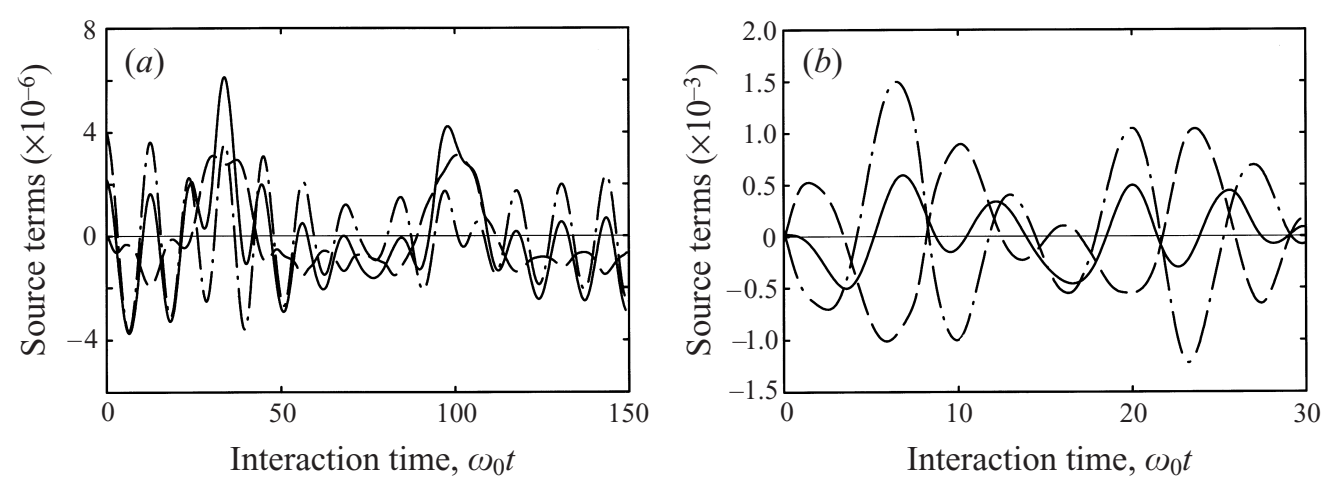

FIGURE 22. (a) Source terms for vortex ring leapfrogging case (cf. figure 4 of Tang \& Ko 1995). (b) Source terms for vortex ring coalescence case (cf. figure 9 of Tang \& Ko 1995). ——, $\sum \ddot{S} ;-\cdot-$, $\sum \ddot{S}_{1}=\sum\left(\partial^{3} / \partial t^{3}\right)\left(\Gamma r_{c}^{2}\left(y_{i c 1}-y_{c 1}\right)\right) ;-\ldots, \sum \ddot{S}_{2}=\sum\left(\partial^{3} / \partial t^{3}\right)\left(\Gamma r_{c}^{2} y_{c 1}^{\prime}\right)$.

\section{Comparisons with existing results}

The results obtained in the present study suggest that the sound from the interactions of two vortex pairs is generated due basically to two mechanisms, regardless of the type of interaction. The first one is due to the jerking and accelerating motions of the vortex centroids, which result in an imbalance of the vortex force in the system. The second one is due to the core deformation, which generates sound of higher frequencies than that generated by the former. The former mechanism is referred to as the vortex centroid dynamics, and the latter as the microscopic vortex core dynamics.

In order to extend the present concept of the separated source terms $\ddot{S}_{1}$ and $\ddot{S}_{2}$ to the case of vortex-ring sound generation, a vortex-ring version of these terms must be developed. Since $\Gamma y_{c 2}$ represents the vortex impulse of a vorticity patch in the longitudinal direction and the vortex impulse of a vortex ring with the same circulation is proportional to $\Gamma r_{c}^{2}$, where $r_{c}$ is the radius of the vortex ring (Pozrikidis 1986), $\ddot{S}_{1}$ and $\ddot{S}_{2}$ for the vortex-ring case are

$$
\dddot{S}_{1}=\sum \frac{\partial^{3}}{\partial t^{3}}\left(\Gamma r_{c}^{2}\left(y_{i c 1}-y_{c 1}\right)\right) \quad \text { and } \quad \dddot{S}_{2}=\sum \frac{\partial^{3}}{\partial t^{3}}\left(\Gamma r_{c}^{2} y_{c 1}^{\prime}\right)
$$

respectively. The definitions of $y_{c 1}$ and $y_{c 1}^{\prime}$ follow those in (3) and (12). For $y_{i c 1}$, the definition of Pozrikidis (1986) is adopted. Some of the results of Tang \& Ko (1995) are re-analysed. It is observed that, for the vortex-ring leapfrogging case, one obtains $\ddot{S}_{1} \approx \sum \Gamma r_{c}^{2}\left(\partial^{3} / \partial t^{3}\right)\left(y_{i c 1}-y_{c 1}\right)$ (not shown here). The wavy oscillations of the overall source strength are due to $\ddot{S}_{1}$, and thus $\left(\partial^{3} / \partial t^{3}\right)\left(y_{i c 1}-y_{c 1}\right)$ (figure 22a). The sound produced by $\ddot{S}_{2}$ is of low frequency and is dominant only at or close to the instants of slip through (figure 22a). This agrees with the results presented in figure $5(c)$. In the case of vortex ring coalescence, almost equal contributions of $\ddot{S}_{1}$ and $\ddot{S}_{2}$, as in figure 19 , are observed (figure 22b), showing that the sound generation mechanisms for the vortex ring and vortex pair interactions are very similar. However, the approximation $\ddot{S}_{1} \approx \sum \Gamma r_{c}^{2}\left(\partial^{3} / \partial t^{3}\right)\left(y_{i c 1}-y_{c 1}\right)$ does not hold in this case as the rate of change of the vortex-ring impulse is comparable to that of $\left(y_{i c 1}-y_{c 1}\right)$ (not shown here). The forms of partial coalescence of vortex rings studied by Tang \& Ko (1995) are not the same as those in the present vortex-pair case. Thus, the corresponding results are not presented. 


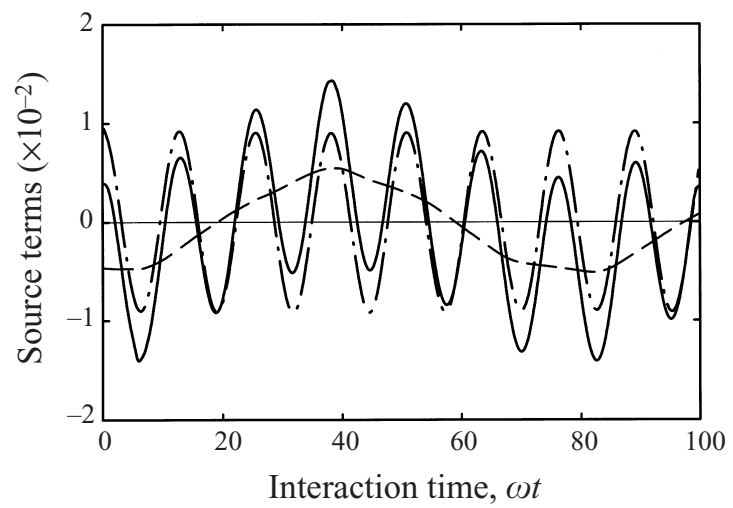

FIGURE 23. Source terms for two-dimensional vortex leapfrogging case (cf. figure 10 of Tang \& Ko 1997). -,$\ddot{S} ;-—, \ddot{S}_{2}=\Gamma\left(\partial^{3} / \partial t^{3}\right)\left(y_{c 2} y_{c 1}^{\prime}\right) ;-\cdot-, \ddot{S}_{1}=\Gamma y_{c 2}\left(\partial^{3} / \partial t^{3}\right)\left(y_{i c 1}-y_{c 1}\right)$.

Wavy oscillations are also observed in the numerical results on the sound generated by the interactions of two identical two-dimensional vortices (Tang \& Ko 1997). The major difference between the work of Tang \& Ko (1997) and the present investigation is that only one lateral quadrupole having directivity $\cos 2 \theta$ exists in the present study. The quadrupole source with directivity $\cos 2 \theta$ of Tang \& Ko (1997) is re-analysed, based on the present derived source terms (equation (12)). Figure 23 is a typical example, which suggests that the source of the wavy oscillations in the leapfrogging case is, again, $\ddot{S}_{1}=\Gamma y_{c 2}\left(\partial^{3} / \partial t^{3}\right)\left(y_{i c 1}-y_{c 1}\right)$, and that the lower frequency one is from the vortex centroid dynamics $\ddot{S}_{2}=\Gamma\left(\partial^{3} / \partial t^{3}\right)\left(y_{c 2} y_{c 1}^{\prime}\right)$. It should be noted that $y_{c 1}=y_{c 1}^{\prime}$ in the case of the two identical two-dimensional vortices system of Tang \& Ko (1997), as it is a non-propagating one. For the coalescence, the time variations of $\ddot{S}_{1}$ and $\ddot{S}_{2}$ resemble those of the present study and are not further discussed. No partial coalescence is found for the two-dimensional vortex system of Tang \& Ko (1997). Wavy oscillations are also observed in the results of Mitchell et al. (1995), but it is hard to compare them with the present results as the time variations of the vortex centroids in Mitchell et al. (1995) are not available. A summary of the sound sources in the different vortex systems is given in table 1 .

The present results also tend to agree with the comment of Laufer \& Yen (1983) and the experimental results of Tang \& Ko (1993), which suggest that the noise from a jet is generated in a region in which the jet structures are under high acceleration. However, it is difficult to isolate the jerks of the flow structures in the sound generation process experimentally, due to their short duration and small magnitudes. Nor can the small core dynamics be visualized, as the cores cannot be clearly defined in the real situation.

\section{Suggestions for further investigations}

Though the types of vortex interactions studied in the present investigation are relevant to the coherent structure dynamics inside the jet shear layers, it is expected that the results obtained are applicable to other flow problems involving these interactions. An example is the interaction between the vortex streets behind cylinders $(\mathrm{Ng}$, Cheng $\&$ Ko 1997). However, the sound energy radiated by vortex interactions is less significant than that from the interaction between vortical structures and the cylinder solid 


\begin{tabular}{|c|c|c|c|}
\hline Vortex system & Major sound sources & Mechanism & Remarks \\
\hline \multirow[t]{4}{*}{ Vortex pairs } & $\Gamma y_{c 2} \frac{\partial^{3}}{\partial t^{3}}\left(y_{i c 1}-y_{c 1}\right)$ & $\begin{array}{l}\text { Rate of change of vortex impulse } \\
\text { imbalance about vortex centroid }\end{array}$ & $\begin{array}{l}\text { Important throughout leapfrogging, partial coalescence } \\
\text { and coalescence } \\
\text { Relates directly to rate of change of vortex core } \\
\text { asymmetry (core deformation) and higher frequency } \\
\text { sound generation in the leapfrogging case }\end{array}$ \\
\hline & $\Gamma y_{c 2} \frac{\partial^{3} y_{c 1}^{\prime}}{\partial t^{3}}$ & $\begin{array}{l}\text { Longitudinal jerking motions of vortex } \\
\text { centroid }\end{array}$ & $\begin{array}{l}\text { Important at or close to slip-through instants during } \\
\text { leapfrogging }\end{array}$ \\
\hline & $3 \Gamma \frac{\partial^{2} y_{c 2}}{\partial t^{2}} \frac{\partial y_{c 1}^{\prime}}{\partial t}$ & $\begin{array}{l}\text { Imbalance in transverse vortex force on } \\
\text { vortex cores when vortices are } \\
\text { accelerating }\end{array}$ & $\begin{array}{l}\text { Important at or close to slip-through instants during } \\
\text { leapfrogging }\end{array}$ \\
\hline & $\Gamma \frac{\partial}{\partial t} \sum\left(\frac{\partial y_{c 2}}{\partial t} \frac{\partial y_{c 1}^{\prime}}{\partial t}\right)$ & $\begin{array}{l}\text { Rate of change of net power developed } \\
\text { by transverse components of vortex } \\
\text { forces }\end{array}$ & $\begin{array}{l}\text { Important in partial coalescence and coalescence, } \\
\text { especially when the vortex cores are seriously deformed }\end{array}$ \\
\hline \multirow[t]{4}{*}{ Vortex rings } & $\Gamma r_{c}^{2} \frac{\partial^{3}}{\partial t^{3}}\left(y_{i c 1}-y_{c 1}\right)$ & $\begin{array}{l}\text { Rate of change of vortex impulse } \\
\text { imbalance about vortex centroid }\end{array}$ & $\begin{array}{l}\text { Important throughout leapfrogging } \\
\text { Related to core deformation } \\
\text { Responsible for high-frequency sound generation in the } \\
\text { leapfrogging case }\end{array}$ \\
\hline & $\Gamma r_{c}^{2} \frac{\partial^{3} y_{c 1}^{\prime}}{\partial t^{3}}$ and $3 \Gamma \frac{\partial^{2} r_{c}^{2}}{\partial t^{2}} \frac{\partial y_{c 1}^{\prime}}{\partial t}$ & $\begin{array}{l}\text { Axial jerking and radial accelerating } \\
\text { motions of the vortex centroid } \\
\text { respectively (Tang \& Ko 1995) }\end{array}$ & $\begin{array}{l}\text { Important at or close to slip-through instants during } \\
\text { leapfrogging }\end{array}$ \\
\hline & $\frac{\partial^{3}}{\partial t^{3}}\left(\Gamma r_{c}^{2}\left(y_{i c 1}-y_{c 1}\right)\right)$ & $\begin{array}{l}\text { Related to core deformation and } \\
\text { imbalance of vortex impulse about } \\
\text { vortex centroid }\end{array}$ & $\begin{array}{l}\text { Important throughout coalescence } \\
\text { Actual physical meaning is not known }\end{array}$ \\
\hline & $\frac{\partial^{3}}{\partial t^{3}}\left(\Gamma r_{c}^{2} y_{c 1}^{\prime}\right)$ & Vortex centroid dynamics & $\begin{array}{l}\text { Important throughout coalescence } \\
\text { Actual physical meaning is not known }\end{array}$ \\
\hline \multirow[t]{3}{*}{$\begin{array}{l}\text { Two-dimensional } \\
\quad \text { vortices }\end{array}$} & $\Gamma y_{c 2} \frac{\partial^{3}}{\partial t^{3}}\left(y_{i c 1}-y_{c 1}\right)$ & $\begin{array}{l}\text { Rate of change of vortex impulse } \\
\text { imbalance about vortex centroid }\end{array}$ & $\begin{array}{l}\text { Same as those for the vortex pair, except that partial } \\
\text { coalescence does not occur in this vortex system }\end{array}$ \\
\hline & $\Gamma \frac{\partial^{3} y_{c 2} y_{c 1}^{\prime}}{\partial t^{3}}$ & Vortex centroid dynamics & $\begin{array}{l}\text { Responsible for the low-frequency noise production } \\
\text { throughout leapfrogging } \\
\text { As important as } \Gamma y_{c 2} \partial^{3}\left(y_{i c 1}-y_{c 1}\right) / \partial t^{3} \text { during coalescence }\end{array}$ \\
\hline & & TABLE 1 . Summary of source terms. & \\
\hline
\end{tabular}


surfaces for low-Mach-number flows (Dowling \& Ffowcs Williams 1983). Therefore, a study on the sound produced by finite core vortices in the presence of solid surfaces is necessary before the present concept can be further generalized.

The method of contour dynamics is applicable to inviscid vortex interaction. While it gives a reasonable description of the initial vortex interaction, it may not be able to handle practical situations where strong vortex interactions result in threedimensionality and significant cross-diffusion. The sound generation mechanisms described in the present study, therefore, may not truly reveal those at the later stages of partial coalescence and coalescence in real flows. The technique of direct numerical simulation (for instance, Mitchell et al. 1995) may be useful for further investigations.

Another important question is that, though the present results tend to agree with those of the jet experiments of Laufer \& Yen (1983) and Tang \& Ko (1993), the motions of the vortex centroids and the impulse centres are difficult to determine experimentally. Direct comparison between the existing experimental results and the present findings is nearly impossible. The method of direct numerical simulations (Colonius et al. 1997; Mitchell et al. 1999) would be able to bridge this gap, and thus is a promising direction for further studies.

\section{Conclusions}

The sound generated by the interactions of a pair of identical inviscid vortex pairs with a common axis is investigated numerically by using the method of contour dynamics and the theory of vortex sound. Three types of interactions, namely leapfrogging, partial coalescence and coalescence, are studied. All are relevant to the coherent structure dynamics inside shear layers. The sound generation process for each is also discussed.

The theory of vortex sound suggests the quadrupole nature of the sound field generated. The present results show that the source can be separated into two basic components: one represents sound generation through the vortex centroid dynamics and the other the microscopic dynamics of the vortex cores. The latter is associated with the uneven distribution of vortex impulse at the vortex centroids, which largely depends on the shapes of the vortex cores. Their relative importance depends on the type of vortex pair interaction.

For the leapfrogging motion, the production of sound via the vortex centroid dynamics is related to the longitudinal jerks and the transverse accelerations of the vortex centroids. However, the sound so generated is only important at the instant when the slip through occurs. Wavy oscillations on the time variations of the source strengths are also observed. These oscillations are found to be the results of the microscopic dynamics of the vortex cores and are associated with the rate of change of vortex core asymmetry. They are important throughout the interaction, though the deformation of vortex cores during the leapfrogging interaction is essentially small.

The vortex pairs exchange part of their core fluid during the partial coalescence process. The two source components are equally important when the core is seriously deformed. The wavy oscillations are produced by the same mechanism as in the leapfrogging case, but the source related to the vortex centroid dynamics has a complicated coupling between the vortex forces, jerks and accelerations of the vortex centroids. The relatively more important source is the rate of change of the total power developed by the vortex forces within the interacting system.

The two mechanisms of sound generation are also equally important in the case of coalescence. When the two vortex pairs coalesce, the spatial separation of the vortex 
centroids is small. The dynamics of the two vortex centroids are nearly mirror images of each other when the effect of the overall steady translating motion of the system is neglected. The sound generated by the jerking motions of the vortex centroids gradually becomes insignificant so that the rate of change of the power developed by the vortex forces becomes the major source of sound. The mechanism related to the microscopic core dynamics remains as important as in the previous interaction cases, suggesting that it is independent of the type of vortex interaction.

An effort is made to apply the present source terms to sound generation by the interactions of two two-dimensional vortices and of two vortex rings. The source terms confirm that the occurrence of wavy oscillations in the vortex interaction cases discussed is due to microscopic vortex core dynamics. The vortex centroid dynamics tend to generate sound of much lower frequencies and are important at or close to the slip-through instants during leapfrogging motions in propagating vortex systems, but make a contribution equal to that of the microscopic vortex core dynamics during coalescence.

This study was partly supported by a research grant from the Committee of Conference and Research Grants, The University of Hong Kong and by a donation from Dr Haking Wong. The computing support from the Hong Kong Polytechnic University is appreciated.

\section{REFERENCES}

ACTON, E. 1976 The modelling of large eddies in a two-dimensional shear layer. J. Fluid Mech. 76, 561-592.

Beavers, G. S. \& Wilson, T. A. 1970 Vortex growth in jets. J. Fluid Mech. 44, 97-120.

Colonius, T., Lele, S. K. \& Moin, P. 1997 Sound generation in a mixing layer. J. Fluid Mech. 330, 375-409.

Crighton, D. G. 1972 Radiation from vortex filament motion near a half plane. J. Fluid Mech. 51, $357-362$.

Crighton, D. G. 1975 Basic principle of aerodynamic noise generation. Prog. Aerospace Sci. 16, 31-96.

Davies, P. O. A. L. 1973 Structure of turbulence. J. Sound Vib. 28, 513-526.

DoAK, P. E. 1998 Fluctuating total enthalpy as the basic generalized acoustic field. Theor. Comput. Fluid Dyn. 10, 115-133.

Dowling, A. P. \& Ffowcs Williams, J. E. 1983 Sound and Sources of Sound. Ellis Horwood.

Dritschel, D. G. 1986 The nonlinear evolution of rotating configurations of uniform vorticity. J. Fluid Mech. 172, 157-182.

Ffowcs Williams, J. E. \& Hawkings, D. L. 1968 Shallow water wave generation by unsteady flow. J. Fluid Mech. 31, 779-788.

Howe, M. S. 1975 Contributions to the theory of aerodynamic sound, with application to excess jet noise and the theory of the flute. J. Fluid Mech. 71, 625-673.

Hussain, A. K. M. F. 1986 Coherent structures and turbulence. J. Fluid Mech. 173, 303-356.

Hussain, A. K. M. F. \& Clark, A. R. 1981 On the coherent structure of axisymmetric mixing layer: a flow visualization study. J. Fluid Mech. 104, 263-294.

JACOBS, P. A. \& Pullin, D. I. 1985 Coalescence of stretching vortices. Phys. Fluids 28, 1619-1625.

Kambe, T. \& Minota, T. 1981 Sound radiation from vortex systems. J. Sound Vib. 74, 61-72.

LAufer, J. \& Yen, T. 1983 Noise generation by a low-Mach-number jet. J. Fluid Mech. 134, 1-31.

Leung, R. C. K., TAng, S. K., Ho, I. C. K. \& Ko, N. W. M. 1996 Vortex pairing as a model for jet noise generation. AIAA J. 34, 669-675.

Lush, P. A. 1971 Measurements of subsonic jet noise and comparison with theory. J. Fluid Mech. 46, 477-500.

Mitchell, B. E., Lele, S. K. \& Moin, P. 1995 Direct computation of the sound from a compressible corotating vortex pairs. J. Fluid Mech. 285, 181-202. 
Mitchell, B. E., Lele, S. K. \& Moin, P. 1999 Direct computation of the sound generated by vortex pairing in an axisymmetric jet. J. Fluid Mech. 383, 113-142.

MöHring, W. 1978 On the vortex sound at low Mach number. J. Fluid Mech. 85, 685-691.

NG, C. W., Cheng, V. S. Y. \& Ko, N. W. M. 1997 Numerical study of vortex interactions behind two circular cylinders in bistable flow regime. Fluid Dyn. Res. 19, 379-409.

Pierrehumbert, R. T. 1980 A family of steady, translating vortex pairs with distributed vorticity. J. Fluid Mech. 99, 129-144.

Powell, A. 1964 Vortex sound theory. J. Acoust. Soc. Am. 36, 177-195.

Powell, A. 1995 Why do vortices generate sound? Trans. ASME: J. Vib. Acoust. 177, 252-260.

Pozrikidis, C. 1986 The nonlinear instability of Hill's vortex. J. Fluid Mech. 168, 337-367.

Pozrikidis, C. \& Higdon, J. J. L. 1985 Nonlinear Kelvin-Helmholtz instability of a finite vortex layer. J. Fluid Mech. 157, 225-263.

Pullin, D. I. 1992 Contour dynamics methods. Ann. Rev. Fluid Mech. 24, 89-115.

Saffman, P. G. 1992 Vortex Dynamics. Cambridge University Press.

Shariff, K., LeOnard, A. \& Ferziger, J. H. 1989 Dynamics of a class of vortex rings. NASA Tech. Memo. TM-102257.

Tang, S. K. \& Ffowcs Williams, J. E. 1998 Acoustic radiation of a vortex approaching a circular cylinder with surface suction. Acustica 84, 1007-1013.

TanG, S. K. \& Ko, N. W. M. 1993 A study on the noise generation mechanism in a circular jet. Trans. ASME: J. Fluids Engng 115, 425-435.

TANG, S. K. \& Ko, N. W. M. 1995 On the sound generated from the interaction of two inviscid coaxial vortex rings moving in the same direction. J. Sound Vib. 187, 287-310.

TANG, S. K. \& Ko, N. W. M. 1997 Sound generation by interaction of two inviscid two-dimensional vortices. J. Acoust. Soc. Am. 102, 1463-1473.

Winant, C. D. \& BRowand, F. K. 1974 Vortex pairing: the mechanism of turbulent mixing layer growth at moderate Reynolds number. J. Fluid Mech. 63, 237-255.

Yamada, H. \& Matsui, T. 1979 Mutual slip-through of a pair of vortex rings. Phys. Fluids 22, $1245-1249$.

Zabusky, N. J., Hughes, M. H. \& Roberts, K. V. 1979 Contour dynamics for the Euler equations in two dimensions. J. Comput. Phys. 30, 96-106. 